\title{
Modeling Mortality Rates Using Heligman-Pollard and Lee-Carter in Nigeria
}

\author{
Yahaya Haruna Umar*, Ugboh Joshua Chukwudi \\ Statistics Department, University of Abuja, Abuja, Nigeria \\ Email address: \\ yahagumau@gmail.com (Y. H. Umar),joshuachucks05@yahoo.com (U. J. Chukwudi) \\ ${ }^{*}$ Corresponding author
}

\section{To cite this article:}

Yahaya Haruna Umar, Ugboh Joshua Chukwudi. Modeling Mortality Rates Using Heligman-Pollard and Lee-carter in Nigeria. American Journal of Theoretical and Applied Statistics. Vol. 8, No. 6, 2019, pp. 221-239. doi: 10.11648/j.ajtas.20190806.14

Received: April 25, 2019; Accepted: September 29, 2019; Published: November 13, 2019

\begin{abstract}
In recent years the need for accurate mortality statistics has been emphasized by researchers in planning, analyzing, monitoring and projection of health situations in the country. It helps the government and other health agencies initiate various programs that can improve life expectancy especially in developing countries like Nigeria. Given the impact of mortality rates on the population size, structure, social security system, life insurance and pension (from actuarial point of view) there is need to understand how mortality patterns change with time. According to past studies the Heligman-Pollard (Henceforth HP) model and Lee-Carter (Henceforth LC) model have been widely accepted and use by researchers in forecasting future mortality. In this study both models were applied to Nigerian data with the objective to investigate the accuracy of their performances by comparing their assumptions. The LC model parameters were estimated based on the singular value decomposition technique (SVD), while HP model parameters were estimated using nonlinear least squares method. Autoregressive Integrated Moving Average (ARIMA) procedure was applied to acquire to forecasted parameters for both models. To investigate the accuracy of the estimation, the forecasted results were compared based on the mean absolute percentage error (MAPE). The results indicate that both models provide better results for female population. However, for the elderly female population, HP model seems to overestimate to the mortality rates while LC model seems to underestimate to the mortality rates. Although the HP model does not seem to follow the pattern of the actual mortality rates, after further analysis was carried out it was discovered that the HP model gave a better forecast than the Lee-Carter model. Based on the HP model the forecasted probabilities of death were used to construct an abridged life table for males and females and the life expectancy at $\mathrm{e}_{0}$ and $\mathrm{e}_{75}$ were obtained. From our results we see that males experience higher life expectancy than females due to the mortality rates experienced by both sexes. Given the level of mortality rate especially in developing countries like Nigeria, the study therefore recommends the need for a vital registration system that could continuously and reliably collect information because it is well known that incomplete data affect the performance of a model to forecast. To ascertain level and pattern of mortality rate especially in adult, parameterization model especially the HP model should be considered because of it lesser errors, with the view of achieving a robust forecasting model that could improve the understanding of the pattern of general mortality rate and how it affects life expectancy level in the country.
\end{abstract}

Keywords: Mortality Rate, Heligman-Pollard Model, Lee-Carter Model, Levenberg-marquardt Iteration Procedures, Nigeria

\section{Introduction}

There has been interest on the future of human survival that has shown a remarkable rise in life expectancy. In developed countries especially countries in Asia, Europe and North-America there has been an improvement in life expectancy which has brought about the further decline in mortality rate, while countries in Africa has experienced slow growth in life expectancy probably because of some social and development significance. Worldwide, the average life expectancy at birth was 70.5 years (68 years and 4 months for males and 72 years and 8 months for females) over the period of 2010-2015 according to United Nations World Population Prospects 2015 Revision. It has been revealed that Women on average live longer than men in all major regions of the world except for Mali and Swaziland. Despite the 
initiative of the World Health Organization (WHO) on safe motherhood the mortality condition in Nigeria has not been dealt with. All effort by the ministry of health to make direct improvement on the health sector in the country has been to no avail. Generally, adult mortality indicators are used to assess the health status of a population, especially in developing country like Nigeria. Estimation of these mortality rates requires complete and accurate data on adult and mortality deaths. Data were collected on the living of respondents' siblings, While early childhood mortality is high and varies substantially according to social and economic development, death rate are much lower at adult ages, and estimates for a subgroups can be distorted by a small sample sizes. Maternal mortality is an aspect of adult mortality that is of a particular interest in the Nigerian context. Worldwide the most countries with the highest maternal mortality ratios are in Africa, and an estimated 14 percent of maternal deaths globally occur in Nigeria according to the United Nations Economic Commission for Africa (UNECA) 2013. According to data from Nigeria's five-Year Countdown Strategy for achieving Millennium Development Goals (MDGs) shows that although maternal mortality fell from 800 deaths per 100,000 live births in 2003 to 545 deaths per 100,000 live births in 2008, progress related to this goal has been slow and still remains a major challenge to the Federal Government of Nigeria. In addition to other interventions designed to reduce maternal mortality and achieve the (MDG) target of 250 deaths per 100,000 live births in Nigeria, the government in collaborations with other development partners, has continued to improve access to quality maternal health services through the Community Health Insurance Scheme. Therefore as a result of this emerging concern, the need for mortality projections has become a growing issue for researchers and among actuaries. The model proposed by Lee and Carter has received so much acceptance because, it produces satisfactory fit and forecast for several countries and has been used for decades by researchers because of its standard framework for projecting future mortality rate in population projections $[1,16]$. It has been observed that the LC model is a simple but highly structured model, where there is little or no allowance for uncertainty. Previous study has shown that Lee-Carter followed the mortality pattern very well for most of the ages $[2,3]$. Population Projections often involves the forecasting of age-specifics fertility and mortality rates, which is a forecasting problem of large dimension. Age-specifics mortality forecast is important to achieve age details in the population projections, to approximate each year's fertility or mortality rates in terms of a set of curve. Parameters are therefore forecasted to produce a fitted curve with some modifications which is the forecasts of the rates. Numerous studies have been carried out on mortality forecast. Over the years there have been remarkable rise in life expectancy in developed countries especially countries in Asia, Europe and North-America because of this, there have been a decrease in mortality rate, while countries in Africa has experienced slow growth in life expectancy probably because of some social and development significance. Significant improvement has been made in mortality forecasting which made the link between pension and changes in life expectancy clearly understood. The monthly pension payments are made based on the remaining life expectancy when people retire. Some countries have linked benefit level to life expectancy. Mortality forecasting is also important in making national policy as fertility rate projections because expected number of death would have a significant bearing on the Nation's objective in transferring resources from one generation to another and also socio-economic-demographic policy decisions. Generation of resources and vision of allocation of resources is being pressured by deaths. The death of old aged people is not as important as the deaths of the young and capable ones and the failure to stop death of the young and capable is simply a failure of a nation's vision and scientific ability. The aspects of age-specific death rate (ASDR) and its patterns is approached by forecasting the mortality rate which is important for demographic reasons and it is important to put the uncertainty of life-expectancy in the forecasting model. Even if the theoretical mortality model is the same, the empirical treatment of error term widely differs. Because of the importance of mortality forecasts, numerous models for mortality modelling and forecasting has been developed. The majority of these models are known to be extrapolative in nature, of which the Lee-Carter has become frequently used. This model summarises the mortality experience by age of a single population over a period of time trend. Although previous studies have documented that both models provides better results for male population, but for elderly female population, HP seems to underestimate the mortality rate while LC seems to overestimate the mortality rate [4, 12]. Again both models were used in modeling and forecasting and cross-validation techniques were used to measure how accurately these two models performed in practice $[5,15]$. The results, based on the MAPE, showed that the HP model seemed to fit better to the Dutch data than the Lee-Carter model. Therefore as a result of this emerging concern, the need for mortality projections has become a growing issue for researchers and among actuaries. Both models have received so much acceptance and for the purpose this study investigate the performance of these two models in forecasting future mortality rates by applying both methods to Nigeria mortality statistics and make mortality projections $[1,6]$.

\section{Data}

Due to the difficulties involved in gathering sufficient data, this study used data extracted from the WHO Indicator and Measurement Registry (IMR). IMR is a central source of metadata of health-related indicator used by WHO and the organizations. This study covers the age specific mortality rate records of people (both males and females) who died from the infant and childhood ages of 0 to 4,5 to 9 and 10-14 years and adult ages of 15 to 85 and above, for the time periods 2000 to 2016 in Nigeria. The Global Health 
Observatory (GHO) data is WHO gateway to health related statistics of its 194 member states. It provides access to over 1000 indicators health topics including mortality and burden of diseases, the Millennium Development Goals (MDG's) child nutrition, child health, maternal and reproductive health, immunization HIV/AIDS, communicable diseases and risk factors etc. Although datasets represents the best estimates of WHO, using methodologies for specific indicators that aim for comparability across countries and time. Updated as revised data becomes available therefore they are not always the same as National estimates. Data files are organized by sex, age and time. Population size is given for one-year and five-year age groups. One year age groups means $1,2, \ldots, 109,110+$; and five-year age groups means 0 , $1-4,5-9,10-14, \ldots, 105-109,110+$. Age groups are defined in terms of actual age, for instance, "10-14" extends from exact age 10 to right before the 15 th birthday. In this project, the data we will use is the death rate period and the population size is five-year age groups. This study involves a statistical analysis carried out to forecast the mortality rate in Nigeria by fitting the HP and LC model on Nigeria data, given the probability of death $\left(q_{x}\right)$ and age-specifics mortality data in Nigeria from $<1$ to $85+$ over a time period of 16 years that is 2000-2016. The model parameters are estimated using the nonlinear least squares and singular value decomposition (SVD) method respectively after which the model parameters for both models were predicted to obtain the forecasted mortality rates. Forecast of the mortality rates was done by applying the Random walk with drift (RWD) model. The analysis of this study was done using the biplot add-in in Microsoft Excel 7, R and SPSS.

\section{Method}

The methods used in this study involve nonlinear equations and traditional singular value decomposition (SVD) in estimating the models parameters of the HeligmanPollard and Lee-Carter model respectively. Also univariate time series method known as the Autoregressive Integrated Moving Average (ARIMA) procedure is applied to acquire the forecasted parameters for both models as the forecasted mortality rates are obtained by using all the values of forecasted parameters. To investigate the accuracy of the estimation, the forecasted results will be compared based on the error analysis.

\subsection{Heligman-Pollard Model}

In 1980L. Heligman and J. H Pollard proposed the first law of mortality which was originally designed in order to eliminate the lack of previous models. It contains eight parameters and tried to describe the entire life span. The model consist of a mathematical formula containing three term, each representing a distinct component of mortality and was firstly fitted to the Australian mortality data. The HP model is an eight parameter model that can give a better fit to an entire mortality curve. It is an example of a parameterisation function also known as the "laws of mortality "which seek to parsimoniously represent the age pattern of mortality. The Heligman and Pollard's first law is mathematically proposed as follows;

$$
\frac{q_{x}}{p_{x}}=A^{(x+B) C}+D \exp \left[-E(\ln x-\ln F)^{2}\right]+G H^{\left(x-x_{0}\right)}
$$

Where;

$q_{x}$ : is the probability that a person at age $x$ will die before attaining age $x+1$ for the observed data. It takes the value between 0 and 1 only. $p_{x}=1-q_{x} A B C D E F G$ and $H$ Are unknown parameters to be estimated by non-linear the weighted least square method. The curve is continuous and applicable over the entire age ranges $0 \leq x<\infty$.

This model consists of three terms, each term representing a distinct component of mortality while capturing: The first term $A^{(x+B) C}$ represents the age pattern of mortality in childhood and represents the childhood mortality. That is parameter $A$ reflects the infant mortality rate; $B$ reflects the mortality rate of 1 year old children i.e. it reflects the difference between $q_{0}$ and $q_{1} ; C$ reflects the fall of mortality. $A, B, C$ takes take a value between or 0 and 1 .

The second term $D \exp \left[-E(\ln x-\ln F)^{2}\right]$ represents the young adulthood, accidental mortality also known as (accidental hump) in the demography literature. The parameter $D$ also measures rate of mortality decline up to early adult life, the severity of the accident hump and takes the value between 0 and $1 . E$ Is the spread; $F$ represents the location. And the third term $G H x$ also called the Gompertz exponential, $G$ reflects the level of Senescent mortality (the exponential pattern of mortality of adult age) and $H$ represents the geometric rise in mortality at older ages, which is between the domain of interval $(0,1)$ and $(0, \infty)$ respectively. A concave downward curve means that the logarithm of mortality rates at older age increases, but at slower rates as age increases. It would indicate that the age pattern of mortality has a global maximum. In contrast, a concave upward curve implies log-mortality rates growing at a faster pace as age increases.

\subsubsection{Merits of Heligman-Pollard Model}

Parametric methods, like the Heligman-Pollard, have several advantages for describing mortality, including smoothness, interpolation, analytic manipulation, parsimony, comparison and trends (Dellaportas, 2001; Debonetal., 2005). Below are other advantages of the Heligman-Pollard model.

i. It model mortality at a fixed point in time for all ages.

ii. Each of the parameters has a straightforward interpretation.

iii. It extracts parameters at a sequence of points in time iv. It is used as a basis for age-period tables.

\subsubsection{Demerits of Heligman-Pollard Model}

The Heligman-Pollard model is usually estimated via least squares methods but instability and over-parameterization - a wide range of parameter estimates producing a similar fit has been revealed to be an issue.

\subsubsection{Assumptions of Heligman-Pollard Model}

i. The model Assumes that because of it relatively few parameters that all parameters have a demographic interpretation. 
ii. Parameters are highly correlated with each other.

iii. The estimates of it parameter gives its value when the square sum $S=\sum x=0\left[\frac{\boldsymbol{q}_{x}}{\boldsymbol{p}_{\boldsymbol{x}}}-1\right]^{2}$ is minimized.

\subsubsection{Levenberg-Marquardt Method}

In this study the standard technique for solving nonlinear least squares problem will be used to get the estimated values of these parameters by using a damped Gauss-Newton iteration procedure [7]. Least squares problems arise in the context of fitting a parameterized function to asset of measured data points by minimizing the sum of the squares of the errors between the data points and the function. If the fit function is not linear in the parameters the least squares problem is nonlinear. The curve fitting method is a combination of two minimization methods: The gradient descent method and the Gauss-Newton method. In the gradient descent method, the sum of squared error is reduced by updating the parameter in the steepest-descent direction while in the Gauss-Newton method, the sum of the squared error is reduced by assuming the least squares function are logically quadratic. The method acts more like a gradient descent method when the parameters are far from their optimal value, and acts like Gauss-Newton method when the parameters are close to their optimal value. It also provides a numerical solution to the mathematical problem of minimizing a sum of squares of several, generally nonlinear functions that depend on a common set of parameters [7].
Like other numeric minimization algorithms, it interpolates between the Gauss-Newton algorithm and the method of gradient descent, which is more robust than the GaussNewton algorithm since in many cases it finds a solution even if it starts very far off the global minimum. It selects the parameter values for the next iteration and this process continues until a preset criterion is met, either the fit has converged or it reaches a preset iteration count limit $[7,13]$. To execute these procedures one has to load the package with the command library (minpack. $l m$ ) in R. This package provides the $\mathrm{R}$ interface to the Levenberg-Marquardt Nonlinear Least-Squares Algorithm and the $\mathrm{nls}$. $l m$ function from the library is used for solving nonlinear least-squares problems by modification of the Levenberg-Marquardt algorithm, with support for lower and upper parameter bounds [8]. Two difficulties in the Gauss-Newton method was addressed [7]. That is; how to update $x^{(k)}$ when columns of $\left(D f\left(x^{(k)}\right)\right.$ are linearly dependent and what to do if the Gauss-Newton update does not reduce $\|f(x)\|^{2}$.

The regularized least squares problem solved in $k$ iteration:

$$
\left\|f\left(x^{(k)}\right)+D f\left(x^{(k)}\right)\left(x-x^{(k)}\right)\right\|^{2}+\gamma^{k}\left\|x-x^{(k)}\right\|^{2}
$$

Solution is given by

$$
x^{(k+1)}=x^{(k)}-\left(D f\left(x^{(k)}\right)^{T} D f\left(x^{(k)}\right)+\gamma^{(k)} I\right)^{-1}\left(D f\left(x^{(k)}\right)^{T} f\left(x^{(k)}\right)\right.
$$

Step $\Delta x^{(k)}=x^{(k+1)}-x^{(k)}$ is

$$
\begin{gathered}
\Delta x^{(k)}=-\left(D f\left(x^{(k)}\right)^{T} D f\left(x^{(k)}\right)+\gamma^{(k)} I\right)^{-1}\left(D f\left(x^{(k)}\right)^{T} f\left(x^{(k)}\right)\right. \\
=\frac{1}{2}\left(D f\left(x^{(k)}\right)^{T} D f\left(x^{(k)}\right)+\gamma^{(k)} I\right)^{-1} \nabla g\left(x^{(k)}\right) \\
\gamma^{(k)}=0 \\
\Delta x^{(k)} \approx-\frac{1}{2 \gamma^{(k)}} \nabla g\left(x^{(k)}\right)
\end{gathered}
$$

In summary

Choose $x^{(1)}$ and $\gamma^{(1)}$ and repeat for $k=1,2, \ldots$

i. Evaluate $f\left(x^{(k)}\right)$ and $A=D f\left(x^{(k)}\right)$

ii. Compute the solution of regularized least squares

$$
\hat{x}=x^{(k)}-\left(A^{T} A+\gamma^{(k)} I\right)^{-1} A^{T} f\left(x^{(k)}\right)
$$

iii. Define $x^{(k+1)}$ and $\gamma^{(k+1)}$ as follows

$$
\begin{gathered}
\left\{x^{(k+1)}=\hat{x} \text { and } \gamma^{(k+1)}=\beta_{1} \gamma^{k} \text { if }\|f(\hat{x})\|^{2}<\left\|f\left(x^{(k)}\right)\right\|^{2}\right. \\
\left\{x^{(k+1)}=x^{(k)} \text { and } \gamma^{(k+1)}=\beta_{2} \gamma^{(k)},\right. \text { otherwise }
\end{gathered}
$$

where; $\beta_{1}$ and $\beta_{2}$ are constants, with $0<\beta_{1}<1<\beta_{2}$.

Terminate if $\nabla g\left(x^{(k)}\right)=2 A^{T} f\left(x^{(k)}\right)$ is sufficiently small.

\subsubsection{Fitting and Applying the Heligman-Pollard Model}

The HP function is fitted to the probability of dying $\left(n q_{x}\right)$ from the abridged life table of the Nigerian population for period 2000, 2003, 2006, 2009, 2012 and 2016 for both male and female. Therefore in order to check if our results follow the Heligman and Pollard law, graphs of $\log \left(\frac{q_{x}}{1-q_{x}}\right)$ was plotted against $x$ for both males and females aged 0 to 90 in year 2000 to 2016 using Microsoft Excel. Both graphs are presented and discussed. The parameters were estimated by minimizing the sum of squared residual using the LevenbergMarquardt iteration procedures which is a modification of Gauss-Newton iteration. The estimates for the parameters obtained through the constrained minimization process are not all within the defined limits. The accident-hump parameter $F$ reaches the upper bound of 100 seven times. Hence, the accident-hump parameters D, E, F and the mortality for older ages parameter $G$ are non-significantly different from zero even at a ten percent significance level. The same is true for the child-mortality parameters A and B for the one year when $B$ reaches the value of one. This issue is well-known and is due to multicollinearity. Indeed, this multicollinearity causes irregular changes of the parameter 
estimates from one year to another even if the mortality rates of adjoining years are similar. The solution usually adopted in the past is to fix the value of some parameters prior to the fitting process. However, since such an approach yields to some loss in the fitting precision and for simplification purpose, some periods were selected for further analysis. Thus after estimation the parameters that give the best fit that is $n l s$ were used.

\subsubsection{Estimation of Heligman-Pollard Parameters}

The parameters of the HP function are estimated by minimizing the sum of squares, by Letting $Q(x ; c)$ to denote the right hand side of 3.10 , it is reasonable to expect that the parameters can be estimated by minimizing the sum of squares

$$
\sum_{x=0}^{85+}\left(\frac{\widehat{q_{x}}}{q_{x}}-Q(x ; c)\right)^{2}
$$

Where; $\widehat{q_{x}}$ is the fitted mortality at age $x$ and $q_{x}$ is the observed mortality. The maximal age included in the model is increased from 94 to 101 in 1970 as data for older ages become more reliable. In order to estimate these parameters, we start by collecting the abridged $n q_{x}$ values which were given in the abridged life tables for the Nigerian population. In the study, the abridged life tables for the Nigerian population in year 2000 until year 2016 for both males and females were collected. Then these values will be fitted to
(1). The fit is judged on the basis of the sum of the squared differences or 'residuals' between the input data points and the function values, evaluated at the same places. Thus, the fit is used to find a set of parameters that 'best' fits the data to user-defined function. A parameter is a user-defined variable that fit will adjust, which is an unknown quantity in the function declaration. However, the sum of squares to be minimized in the study was given by

$$
S^{2}=\sum_{x}\left(\frac{\widehat{q_{x}}}{q_{x}}-1\right)^{2}
$$

Often the function to be fitted will be based on a model or theory that attempts to describe or predict the behavior of the data. Then 'fit' can be used to find values for free parameters of the model, to determine how well the data fits the model, and to estimate a standard error for each parameter. In the study, the function to be fitted will be based on (1). Several algorithms exist to solve non-linear equations and many languages and environments for statistical computing contain such functionality. A non-linear minimization requires initial estimations for the parameters. Starting values need to be as precise as possible for the algorithm to converge to an optimum, which may lead to some difficulties. By plotting the HP function on the same graph with the actual mortality, one can read its initial value. Below is the nonlinear least squares algorithm using the (minpack. lm) in $\mathrm{R}$.

$$
\begin{aligned}
& \text { library (minpack. lm) } \\
& \text { \#\# valuesoverwhichtosimulatedata } \\
& x<-\operatorname{seq}(0,5, \text { length }=100) \\
& \text { \#\# modelbasedonalistofparameters } \\
& \text { getPred }<- \text { function }(\operatorname{par} S, x x) \operatorname{par} S \$ a * \exp (x x * \operatorname{par} S \$ b)+\operatorname{par} S \$ c \\
& \text { \#\# parametervaluesusedtosimulatedata } \\
& p p<-\operatorname{list}(a=9, b=-1, c=6) \\
& \text { \#\# simulateddata,withnoise } \\
& \operatorname{simDNoisy}<-\operatorname{getPred}(p p, x)+\operatorname{rnorm}(\operatorname{length}(x), s d=.1) \\
& \text { \#\# plotdata } \\
& \operatorname{plot}(x, \operatorname{sim} \text { Noisy, } \text { main }=\text { "data") } \\
& \text { \#\# residualfunction } \\
& \text { residFun }<- \text { function }(p, \text { observed, } x x) \text { observed }-\operatorname{getPred}(p, x x) \\
& \text { \#\# startingvaluesforparameters } \\
& \text { parStart }<-\operatorname{list}(a=3, b=-.001, c=1) \\
& \text { \#\# performfit } \\
& \text { nls. out }<-n l s . \operatorname{lm}(\text { par }=\text { parStart }, f n=\text { residFun, observed }=\text { simDNoisy, } \\
& x x=x, \text { control }=\text { nls.lm } \cdot \operatorname{control}(\text { nprint }=1))
\end{aligned}
$$


lines $(x, \operatorname{getPred}(\operatorname{as} . \operatorname{list}(\operatorname{coef}(n l s . o u t)), x), \operatorname{col}=2, \operatorname{lwd}=2)$

\section{\#\# summaryinformationonparameterestimates}

summary(nls.out).

\subsubsection{Forecasting the Parameters of Heigman-Pollard Model}

The forecast of the parameters of (1) model was done using method in (31) using SPSS. Constants and outliers from the fitted parameters were removed for smooth forecasting. All parameters for selected periods were forecasted and plugged back into (1) to determine the forecasted mortality rates.

\subsection{Lee-Carter Model}

The model was introduced by Ronald D. Lee and Lawrence Carter in 1992 with the article modelling and forecasting the time series of U.S mortality (Journal of the American Statistical Association 87 September) 659-671; The Lee-Carter model is a demographic model that involves statistical time series techniques and it is a model of agespecifics death rate with a dominant time series component having a relative age component that is fixed with a time series model (ARIMA). The model was firstly applied to the United State death rate data for year 1933-1987 which was said to perform well in generating the output since then the Lee-Carter model has been widely used for demographic and actuarial applications in various countries and has been modified into various extensions. The method describes the $\log$ of a time series of age-specific death rates as the sum of an age-specific component that is independent of time and another component that is the product of a time-varying parameter reflecting the general level of mortality, and an age-specific component that represents how rapidly or slowly mortality at each age varies when the general level of mortality changes. Lee-Carter model is one of the most popular methods for modeling mortality rates for all ages, because it is easily applied and provides fairly accurate mortality estimations and population projections. It became reference and leading statistical model for forecasting mortality. The model combines a demographic model with statistical model time series to forecast mortality. The LC model is a simple model comprising of two independently linear variables $x$ representing (age) and $t$ representing (time) or period (in Calendar year). And it is defined as

$$
\ln m_{x, t}=\alpha_{x}+\beta_{x} k_{t}+\varepsilon_{x, t}
$$

Where,

$m_{x, t}=\frac{d_{x, t}}{e_{x, t}}$, denotes the matrix of death rate on a specific age group at age $(x)$.

$\left(x=x_{1}, \ldots, x_{n}\right)$ Over a Period $(t)\left(t=t_{1}, t_{1}+1,+T-\right.$ 1 ), It is the ratio of total deaths to the population at risk and also called the central mortality rate at age $x$ in year $t$.

$\alpha_{x}$; denotes the empirical average (overtime) pattern of the logarithm mortality rate describing the overall mean of the age profile. $\beta_{x}$; denotes the sensitivity rate to change at agex to the time trend i.e (it represents the relative rate at which an individual at agex will respond to Change in the overall level of mortality overtime).

$k_{t}$; denotes the varying time trend index of general mortality level, It tells if change at a specific age occurs faster than the main trend itself.

$\varepsilon_{x, t}$; denotes the residual or a set of random error (white noise) that gives the age specific factors not captured in the model. It is Gaussian distributed that is each are independently identically distributed that assumes.

$$
\varepsilon_{x, t} \sim N\left(0, \sigma^{2}\right) E\left(\varepsilon_{x, t}\right)=0, \operatorname{var}\left(\varepsilon_{x, t}\right)=\sigma^{2}
$$

Interpretation of parameters (using sets of Constraint)

$$
\begin{array}{r}
\alpha_{x}=\frac{1}{T} \sum_{t} \ln m_{x, t} \\
\beta_{x}: \frac{d}{d t} \ln m_{x, t}-\beta_{x} \frac{d}{d t} k_{t} \\
k_{t}=\sum_{t}\left(\ln m_{x, t}-\alpha_{x}\right)
\end{array}
$$

The equation (12) is the evolution over time of mortality. Because the equation underpinning the Lee-Carter model is considered to be over parameterized due to the log-bilinear multiplicative terms $\beta_{x} k_{t}$, Parameters are constrained by $\sum_{x} \beta_{x}=1$ and $\sum_{t} k_{t}=0$ to ensure it is identifiable.

\subsubsection{Merits of Lee-Carter Model}

i. It produces a satisfactory fit and efficient in forecasting adult mortality.

ii. It produces high confidence to extrapolations because it makes mortality trends linear.

iii. It gives more reasonable estimates of forecast.

iv. It out performs other models with respect to its prediction errors.

v. It is reserved in numbers of parameters to be used.

vi. The method involves minimal subjective judgement and produces stochastic forecast with probabilistic prediction intervals.

\subsubsection{Demerits of Lee-Carter Model}

The disadvantage of the Lee-Carter model is the constant assumption for the parameters and the limiting mortality of zero. This issue has prompted lots of discussions and many proposed modification. And these assume that some parameters are constant over time, whereas empirical studies in various countries do not support this assumption time. Nevertheless, there are still some limitations to the standard Lee-Carter model. The model does not work well to forecast mortality for a group of populations 36, and it cannot deal with limited data, also it cannot include external factors which have impacts on mortality and it has narrow prediction intervals. 


\subsubsection{Assumptions of Lee-Carter Model}

i. The Lee-Carter model assumes that the dynamics of death rate is driven by a single time-varying parameter.

ii. It assumes that the ratio of the rates of mortality change, at different ages remains constant over time (invariant $\beta_{x}$ ).

iii. It assumes a constant error variance across ages.

iv. It assumes that the residual also known as the random error term $\varepsilon_{x, t}$ are normally distributed that is $\varepsilon_{x, t} \sim N\left(0, \sigma^{2}\right) E\left(\varepsilon_{x, t}\right)=0, \operatorname{var}\left(\varepsilon_{x, t}\right)=\sigma^{2}$.

v. It also assumes that $\alpha_{x}$ and $\beta_{x}$ are vectors of age specifics and are constant overtime $(t)$ for all ages $(x)$.

vi. Sex is measured separately and jointly using $k_{t}$ but two different sets of $\alpha_{x}$ and $\beta_{x}$.

\subsubsection{Development of Lee-Carter Model}

The model has undergone different extensions and modifications added to improve the performance of the model by adding additional statistical features as non parametric smoothing. Previous extension by Carter (1995), Lee and Miller (2001), other two extensions including a cohort based extension to the Lee-Carter model for mortality reduction factors $[9,14]$. Lee-carter has been applied to cause of death data to sex separately and by age [1]. A state space model is used to summarize the model's development, extensions and applications as stochastic forecast of Social Security system finances.

\subsubsection{Fitting and Applying Lee-Carter Model}

To ensure good performance, we decide an appropriate method to estimate the model's parameters and it has to be done carefully at the very beginning. In their original paper, Lee and Carter (1992) applied a two-stage estimation procedure. In the first stage, singular value decomposition (SVD) is applied to the matrix of $\log \left(m_{x, t}\right)-\alpha_{x}$ to obtain estimates of $\beta_{x}$ and $k_{t}[10]$. Then in the second stage, the time series of $k_{t}$ is re-estimated by the method of so called" second stage estimation". Lee and Carter noticed that once $\beta_{x}$ and $k_{t}$ have been estimated, the observed total number of deaths $D_{t} \equiv \sum_{x} D_{x, t}$ is not guaranteed to be equal to the fitted number of deaths. Therefore, they made a second stage estimation of $k_{t}$ by finding a value that makes the observed number of deaths equal to the predicted number of deaths. That is, they searched for $k_{t}$ such that

$$
D_{t}=\sum_{x}\left\{\exp \left(\alpha_{x}+\beta_{x} k_{t}\right) N_{x, t}\right\}
$$

Where,

$D_{t}$ is the total number of deaths in year t;

$N_{x, t}$ is the population (exposure to risk) of age $x$ in year $t$.

However, different criteria have been proposed for this method. SVD is the best alternative for estimating the mortality index $k_{t}$. Another advantage of SVD is that it can be easily facilitated by using Biplot* in Microsoft Excel. In this study, we skip the re-estimation stage and apply the first stage SVD method directly.

\subsubsection{Estimating the Lee-Carter Parameters}

The Lee-Carter model unlike most regression formulations is not a common model structure with observed variables on the right-hand side and thus it cannot be fit with simple regression formulations. Hence, the estimation of $\beta_{x}$ and $k_{t}$ cannot be solved explicitly. The (SVD) is a method for transforming correlated variables into a set of uncorrelated ones by exposing the various relationships among the original data items. It is based on a theorem from linear algebra which says that a matrix A can be broken down into the product of three matrices - an orthogonal matrix $U$, a diagonal matrix $\mathrm{D}$, and the transpose of an orthogonal matrix $\mathrm{V}$. In other words, any real $m \times n$ matrix $A$ can be decomposed uniquely as:

$$
A=U D V^{T}
$$

$U$ is $m \times n$ and orthogonal (its columns are eigenvectors of $A A^{T}$ )

$$
U^{T} U=I
$$

$V$ is $n \times n$ and orthogonal (itscolumns are eigenvectors of $\left.A^{T} A\right)$

$$
V V^{T}=I
$$

$D$ is $n \times n$ diagonal (non-negative real values called singular values) $D=\operatorname{diag}\left(\rho_{1}, \rho_{2}, \ldots, \rho_{n}\right)$ ordered so that $\rho_{1} \geq \rho_{2} \geq \cdots \geq \rho_{n}$ (if $\rho$ is a singular value of $A$, its square is an eigenvalue if $A^{T} A$ ) Transform the forecast of an agespecific vector $\ln m_{x, t}$ in to forecasting as scalar $k_{t}$ with small error by applying the Singular Value Decomposition (SVD) [10].

From the Lee-Carter model the parameter vector of $\alpha_{x}$ can be easily computed as the average over time of the logarithm of the central death rate. Then we apply the Singular Value Decomposition to matrix.

$$
Z_{x, t}=\ln \left(m_{x, t}\right)-\widehat{\alpha_{x}}
$$

Resulting to matrices

$$
U L V^{\prime}=S V D\left(Z_{x, t}\right)=L_{1} U_{X 1} V_{t 1}+\cdots+L_{X} U_{x X} V_{t X}
$$

Approximation to the first term gives the estimates $\hat{\beta}_{x}=U_{X 1}$ and $k_{t}=L_{1} V_{t 1}$ In applying the singular value decomposition (SVD) six basic steps are required after obtaining logarithm of $m_{x, t}$ of the mortality rate.

$$
\widehat{\alpha_{x}}=\frac{1}{T} \sum_{t=t_{1}}^{t n} \ln \left(m_{x, t}\right)
$$

Create a matrix $Z_{x, t}$ for estimating $\beta_{x}$ and $k_{t}$.

Where;

$$
Z_{x, t}=\ln \left(m_{x, t}\right)-\widehat{\alpha_{x}}=\beta_{x} k_{t}
$$

Apply (SVD) to matrix $Z_{x, t}$ which decomposes into the products:

$$
U L V^{\prime}=S V D\left(Z_{x, t}\right)=L_{1} U_{X 1} V_{t 1}+\cdots+L_{X} U_{x X} V_{t X}
$$

Where; $U$ is the age component, $L$ is the singular values and $V$ representing the time component 
Select SVD Dialog from Biplot in Ms excel, $k_{t}$ is derived from the first vector of the time-component matrix and the first singular value $\left(\widehat{k}_{t}=L_{1} V_{t 1}\right)$ and $\beta_{x}$ is derived from the first vector of age-component matrix $\left(\widehat{\beta_{x}}=U_{X 1}\right)$.

(Lee-Carter) Approximate a new matrix $Z_{x, t}$ by the product of the estimated parameters $\beta_{x}$ and $k_{t}$ which gives $Z_{x, 1} t_{1}=$ $\beta_{x 1} k_{t 1}$

$$
\begin{gathered}
Z_{x, t}=\left[\begin{array}{llll}
Z_{x 1} t_{1} & Z_{x 1} t_{2} & \ldots & Z_{x 1} t_{n} \\
Z_{x 2} t_{1} & Z_{x 2} t_{2} & \ldots & Z_{x 2} t_{n} \\
\cdots & \cdots & \cdots \\
Z_{x \wedge} t_{1} & Z_{x \wedge t_{2}} & Z_{x_{\wedge} t_{n}}
\end{array}\right] \beta_{x} \\
k_{t} \\
\text { Where } \beta_{x}=\left(\begin{array}{l}
\beta_{1} \\
\beta_{2} \\
\cdots \\
\beta_{n}
\end{array}\right) \\
k_{t}=\left[\begin{array}{llll}
k_{0} & k_{1} & \cdots & k_{n}
\end{array}\right]
\end{gathered}
$$

Estimate the logarithm of the central death rate.

The Singular value decomposition (SVD) which was applied to the log of mortality.

$\ln m_{x, t}-\alpha_{x}=\beta_{x} k_{t} \quad$ Adding constraint $\sum \beta_{x}=1$ and $\sum k_{t}=0$

$\alpha_{x}$ Is computed as average of mortality ratio over time $t$ :

$$
\begin{gathered}
\text { Let } Q=(\alpha, \beta, k)=\sum x, t\left(\ln m_{x, t}-\alpha_{x}-\beta_{x} k_{t}\right)^{2} \\
\text { Let } \frac{\partial Q}{\partial \alpha_{x}}=\frac{\partial Q}{\partial \beta_{x}}=\frac{\partial Q}{\partial k t}=0 \\
\frac{\partial Q}{\partial \alpha_{x}}=2 \sum_{t}\left(\ln m_{x, t}-\alpha_{x}-\beta_{x} k_{t}\right)=0 \\
\frac{\partial Q}{\partial \beta_{x}}=2 \sum_{t}\left(\ln m_{x, t}-\alpha_{x}-\beta_{x} k_{t}\right) k_{t}=0 \\
\frac{\partial Q}{\partial k_{t}}=2 \sum_{t}\left(\ln m_{x, t}-\alpha_{x}-\beta_{x} k_{t}\right) \beta_{x}=0
\end{gathered}
$$

Then we say,

$$
\begin{gathered}
\sum_{t} \alpha_{x}=\sum_{t} \ln m_{x, t}-\sum_{t} \beta_{x} k_{t}=\sum_{t} \ln m_{x, t} \\
\alpha_{x}=\frac{1}{T} \sum_{t} \ln m_{x, t}
\end{gathered}
$$

Now let's take a set of new matrix $Z_{x, t}=\ln m_{x, t}-\alpha_{x}$ the equation for $\beta_{x}$ and $k_{t}$

$$
\begin{gathered}
\sum_{t} Z_{x, t} k_{t}=\sum_{t}\left(\ln m_{x, t}-\alpha_{x}\right) k_{t} \\
=\sum_{t}\left(\beta_{x} k_{t}\right) k_{t}=\beta_{x} \sum_{t} k_{t}{ }^{2} \\
\sum_{t} Z_{x, t} \beta_{x}=\sum_{x}\left(\ln m_{x, t}-\alpha_{x}\right) \beta_{x} \\
=\sum_{x}\left(\beta_{x} k_{t}\right) \beta_{x}=k_{t} \sum_{x} \beta_{x}{ }^{2}=k_{t}
\end{gathered}
$$

Since $\sum_{x} \beta_{x}^{2}=1$.

Consider the vector form with $\sum_{t} k_{t}^{2}=b$.
Now using the equation $\left(\sum_{t} k_{t}^{2}-b\right)^{2}=\sum_{x}\left(\sum_{t} k_{t} Z_{x, t}\right)^{2}$. Taking the square root of both sides we have $\sum_{t} k_{t}{ }^{2}-b=$ $\sqrt{\sum_{x}\left(\sum_{t} k_{t} Z_{x, t}\right)^{2}}$

$$
b=\sum_{t} k_{t}^{2}-\sqrt{\sum_{x}\left(\sum_{t} k_{t} Z_{x, t}\right)^{2}}
$$

But inserting $b$ into $\beta_{x}=\left(\sum_{t} k_{t}^{2}-b\right)=\sum_{t} k_{t} Z_{x, t}$ We have:

$$
\begin{gathered}
\beta_{x}\left(\sum_{t} k_{t}^{2}-\sum_{t} k_{t}^{2}+\sqrt{\sum_{x}\left(\sum_{t} k_{t} Z_{x, t}\right)^{2}}\right)=\sum_{t} k_{t} Z_{x, t} \\
\beta_{x}=\frac{\sum_{t} k_{t} z_{x, t}}{\sqrt{\sum_{x}\left(\sum_{t} k_{t} z_{x, t}\right)}} \forall x
\end{gathered}
$$

Hence;

$$
\alpha_{x}=\frac{1}{T} \sum_{t} \ln m_{x, t}, \beta_{x}=\frac{\sum_{t} k_{t} Z_{x, t}}{\sqrt{\sum_{x}\left(\sum_{t} k_{t} Z_{x, t}\right)}}, k_{t}=\sum_{x} \beta_{x} Z_{x, t}
$$

\subsubsection{Forecasting Mortality Index}

In forecasting mortality index, after all estimated parameters have been obtained a possible forecast on mortality rates is carried out by a standard univariate time series model $\operatorname{ARIMA}(0,1,0)$ despite various techniques and testing of other ARIMA specification the Lee-Carter saw that a random walk with drift is most suitable for modeling the mortality index even though other ARIMA model can be used for different data sets the RWD has been used exclusively in practice. The model is given by

$$
k_{t}=k_{t-1}+\theta+\varepsilon_{t}
$$

Where;

$\theta:$ is drift parameter.

$\varepsilon_{t}$ : is an error term with zero mean and constant variance $\varepsilon_{t} \sim N\left(0, \sigma^{2}\right)$ With drift parameter $\hat{\theta}=\frac{\widehat{k_{T}}-\widehat{k_{1}}}{T-1}$.

It shows that $\hat{\theta}$ only depends on the first and last of $k_{t}$ estimates. The drift $\theta$ is estimated with uncertainty and standard error of it estimates is used to form a more complete measure of uncertainty in forecasting $\widehat{k}_{t}$. Then to forecast two periods ahead, we just substitute for the definition of $\widehat{k_{t-1}}$ which is $m_{x, t+1} \widehat{e^{\widehat{\alpha_{x}}}+\widehat{\beta_{x}} \widehat{k_{t}}}$ moved back in time one period that is;

$$
\begin{gathered}
\widehat{k_{t}}=\widehat{k_{t-1}}+\hat{\theta}+\varepsilon_{t} \\
=\left(\widehat{k_{t-2}}+\hat{\theta}+\varepsilon_{t-1}\right)+\hat{\theta}+\varepsilon_{t} \\
=\widehat{k_{t-2}}+2 \hat{\theta}+\left(\varepsilon_{t-1}+\varepsilon_{t}\right)
\end{gathered}
$$

To forecast $\widehat{k}_{t}$ at time $T+(\Delta t)$ with data available up to period $\mathrm{T}$, we follow the same procedure and iterate $(\Delta t)$ times and obtain;

$$
\begin{gathered}
\widehat{k_{T+(\Delta t)}}=\widehat{k_{T}}+(\Delta t) \hat{\theta}+\sum_{n}^{(\Delta t)} \varepsilon_{T+n-1} \\
=\widehat{k_{T}}+(\Delta t) \hat{\theta}+\sqrt{(\Delta t) \varepsilon_{t}}
\end{gathered}
$$


By ignoring the error term, we can obtain forecast point estimates, which follow a straight line as a function of $(\Delta t)$ with slope $\hat{\theta}$ :

$$
\begin{aligned}
& \widehat{k_{T+(\Delta t)}}=\widehat{k_{T}}+(\Delta t) \hat{\theta} \\
& =\widehat{k_{T}}+(\Delta t) \frac{\widehat{k_{T}}-\widehat{k_{1}}}{T-1}
\end{aligned}
$$

In forecasting $\widehat{k}_{t}$ we extrapolate from a straight line drawn through the first $\widehat{k_{1}}$ to the last $\widehat{k_{T}}$ points and all other $\widehat{k_{t}}$ points are ignored. We plug this expression into the equation (31) to make a point forecast for $k_{t}$.

\subsection{Various Forecast Performance Measures}

It is important we discuss about the commonly used performance measures each of such has it unique properties which makes them different. We consider more than one criterion so as to obtain direction of overall forecast.

\subsubsection{Sum of Squared Error or Residual}

$$
\text { SSEorSSR }=\sum_{x, t}{\widehat{\varepsilon_{x, t}}}^{2}
$$

The properties of SSE or SSR are:

i. It measures the total squared deviation of forecasted observations, from the actual ones.

ii. The properties of SSE are same as those of MSE.

\subsubsection{Mean Absolute Error}

$$
M A E=\frac{1}{A} \sum_{x, t}\left|\widehat{\varepsilon_{x, t}}\right|
$$

The properties are:

i. It measures the average absolute deviation of forecasted values from original ones.

ii. It is also known as the Mean Absolute Deviation (MAD).

iii. It shows the magnitude of overall error, occurred due to forecasting.

iv. The effects of positive and negative errors do not cancel out.

v. For a good forecast, the obtained MAE should be as small as possible.

\subsubsection{Mean Absolute Percentage Error}

$$
M A P E=\frac{1}{A} \sum_{x, t}\left|\frac{\widehat{\varepsilon x, t}}{m_{x, t}}\right|
$$

The properties of MAPE are:

i. This measure represents the percentage of average absolute error occurred.

ii. It is independent of the scale of measurement, but affected by data transformation.

iii. It does not show the direction of error.

iv. In this measure, opposite signed errors do not offset each other.

Where;

$$
\varepsilon_{x, t}=m_{x, t}-\widehat{m_{x, t}}
$$

$$
\begin{aligned}
& x=1,2,---, A \\
& t=1,2,---, T
\end{aligned}
$$

\subsubsection{Model Selection Criteria}

The models are estimated using $\operatorname{ARIMA}(0,1,0)$ RWD and compared based on the mean absolute percentage error (MAPE) after comparison are made model with the least value for these criteria across the error distribution is adjudged the best fitted.

\subsubsection{Forecast Evaluation}

On the Predictive ability the both models, the model selection criterion that will be used to evaluate the predictive ability of the competing models is MAPE but other criteria are not over looked to justify the result.

\subsection{Construction of Abridged Life Table}

The construction of an abridged life table was done using available data which are in the age groups of five-years apart from age 0-1 years and 1-4 years up to a maximum of 85+ age interval. The abridged life table presented in Appendix $i$ are constructed from the Mortality Laws manual (pg8) [11] using the following arguments. $85+$

Age $\mathrm{x}$ years-representing the age groups $0-1,1-4,5-10, \ldots$,

Length of the interval of age intervals $n$.

Average fraction of life lived in age interval $x$ to $x+n$ for the deceased, $\mathrm{n} q_{x}$.

Forecasted central death rates, $n m_{x}$.

Probability of death within $\mathrm{n}$ years, $\mathrm{n} q_{x}$.

Starting cohort number alive radix to 100,000 .

Number of death, $\mathrm{n} d_{x}$.

Number of person years lived in the range $\mathrm{n} L_{x}$.

Aggregate number of years lived by individuals in cohort at start of the interval, $T_{x}$.

Expectation of life, life expectancy $e_{x}$.

From our projected mortality rates, we can build projected life tables and compute the life expectancy at birth and old adult ages after obtaining the predicted probabilities of death $q_{x}$. Let $f_{x}$ be the average number of years lived within the age interval $[x, x+1)$ for people dying at that age. We assume that $f_{x}=\frac{1}{2}$ for all age group except age 0 . For $x=0$, $1-4,5-9, \ldots \ldots, 85+$ To complete the life table calculation, let $p_{x}$ be the probability of surviving from age $x$ to $x+1$. Therefore,

$p_{x}=1-q_{x}$, for all five-year age groups up the age $85+$ from $q_{x}$ making arbitrary $l_{0}$ equal to 100000 the life table is constructed by working down the column of $l$ 's and $d$ 's, applying the recurrence equation:

$$
\begin{gathered}
l_{x+w x}=l_{x}\left(1-q_{x}\right), x=x_{0,} x_{1, \ldots,} x_{k+2} \\
d_{x}=l_{x}-l_{x+w x}=l_{x} q_{x}, x=x_{0,} x_{1, \ldots,} x_{k+2}
\end{gathered}
$$

Where; $l_{x}$ indicates the number of survivors and $d_{x}$ is the distribution of deaths by age in the life table population. The person-years lived by the life-table population in the age interval $[x, x+1)$ are 


$$
L_{x}=w_{x}\left(l_{x} f_{x}^{\prime} d_{x}\right), x=x_{0}, x_{1, \ldots,} x_{k+2}
$$

The person-years remaining for individuals of age $x$ equal

$$
T_{x}=\sum_{x=x 1}^{x k-1} L_{x}
$$

Imply that life expectancy is given by

$$
e_{x_{i}}=\frac{T_{x_{i}}}{l_{x_{i}}}
$$

\section{Statistical Data Analysis and Discussion}

This section presents the statistical analysis of our findings.

\subsection{Heligman-Pollard Model}

Table 1. Result of Estimated Parameters of the simplified H-P model for selected periods 2000, 2003, 2006, 2009, 2012 and 2016 for Males.

\begin{tabular}{llllllll}
\hline Parameters & B & C & D & E & F & H \\
\hline 2000 & 0.00005 & 0.03019 & 0.00233 & -0.7213 & 10.12893 & 1.19436 \\
2003 & 0.00003 & 0.06897 & 0.00201 & -0.7569 & 9.75098 & 1.20968 \\
2006 & 0.00007 & 0.05748 & 0.00196 & -0.7399 & 9.73459 & 1.20269 \\
2009 & 0.00006 & 0.06983 & 0.00193 & -0.7233 & 9.71967 & 1.625198 \\
2012 & 0.00008 & 0.06845 & 0.00170 & -0.7424 & 9.48667 & 1.19645 \\
2016 & 0.00110 & 0.05239 & 0.00169 & -0.7121 & 9.43659 & 1.20387 \\
\hline
\end{tabular}

Table 2. Result of Estimated Parameters of the simplified H-P model for selected periods 2000, 2003, 2006, 2009, 2012 and 2016 for Females.

\begin{tabular}{lllllll}
\hline Parameters & B & C & D & E & F & H \\
\hline 2000 & 0.00014 & 0.03394 & 0.00323 & -0.5686 & 11.15860 & 1.18178 \\
2003 & 0.00017 & 0.04467 & 0.00317 & -0.5692 & 10.79818 & 1.18592 \\
2006 & 0.00068 & 0.03678 & 0.00310 & -0.5519 & 10.75355 & 1.18540 \\
2009 & 0.00061 & 0.06308 & 0.00279 & -0.5639 & 10.51823 & 1.563574 \\
2012 & 0.00081 & 0.05901 & 0.00249 & -0.5804 & 10.14549 & 1.19610 \\
2016 & 0.00143 & 0.02316 & 0.00247 & -0.5469 & 1.3516 & 1.20647 \\
\hline
\end{tabular}

The tables 1 and 2 gives parameters estimated by fitting the model to data for Nigeria as a whole for males and females for periods 2000, 2003, 2006, 20092012 and 2016 respectively. And from our results it has been observed that parameters $B$ which represents the mortality rate of 1 year old children increases as the period increases for both sexes, meaning the mortality rate of a year old child is increasing with time. Parameter $\mathrm{C}$ which represents the decline in childhood mortality has been a fluctuating one for both male and female over selected periods with 2016 having the lowest estimate. Parameters D and F which represent the rate of mortality decline up to early adult life and the location respectively have been declining with time for both sexes and parameter $\mathrm{E}$ which gives the spread of young adult mortality has been constant overtime indicating the slow fall in the accidental hump. Also looking at the results we see that the parameters estimates for females are higher than that of males. Then by inserting these parameters derived during the process into (1), the mortality rates at age $x$ can be calculated. In fitting one set of the HP model for year 2000 for instance, the obtained equation for fitting nonlinear regression equation for males and females, respectively will be as follows

$$
\left.\left.\frac{q_{x}}{p_{x}}=0^{(x+0.00005)^{0.03019}}+0.00233 * \exp \left(-(-0.72126) *\left(\log \left(\frac{x}{10.12890}\right)\right)^{2}\right)+(0) * 1.194366^{\wedge} x\right)\right)
$$

And

$$
\left.\left.\frac{q_{x}}{p_{x}}=0^{(x+0.00014)^{0.03394}}+0.00323 * \exp \left(-(-0.56855) *\left(\log \left(\frac{x}{11.15860}\right)\right)^{2}\right)+(0) * 1.18178^{\wedge} x\right)\right)
$$

Based on the results obtained from the equation above, graphs of mortality rate $\left(q_{x}\right)$ against age $(x)$ for both genders was plotted for comparison purpose see figures 1 and 2 . As seen in figures 1 and 2 we found out that big error occurred at age 0 except for age 1 which show a high mortality rate and shows a declining trend as it reaches ages 5-10. Also we see that females exhibit higher mortality rates than males at all ages, since it increases with age it is generally accepted. We also see that starting from age 15 to 90 mortality rates has increased following a straight line except for ages 10-14. Most mortality research clearly indicates that female mortality is significantly lower than that of male and the difference may be increasing until it reaches the oldest age categories.

From this analysis we see that female has experienced a higher mortality rates which might be due to high morbidity or sickness rate than in males in Nigeria. The fitted HP mortality rate is presented below in table 3 . 


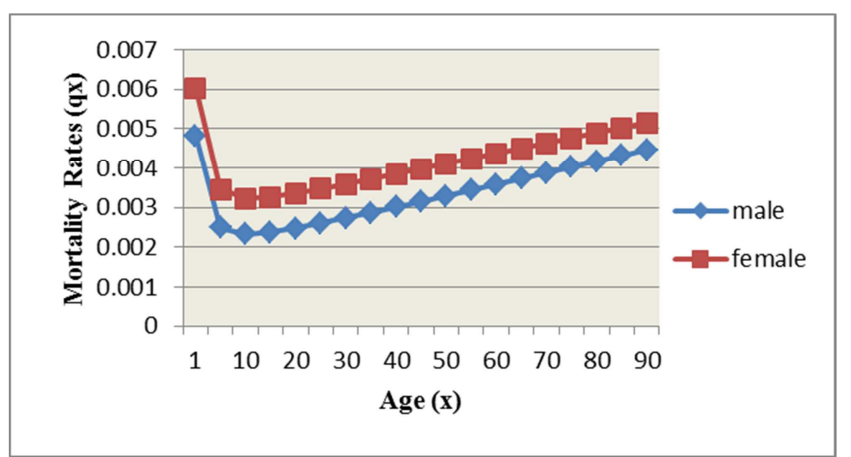

Figure 1. Graph Mortality Rate against Age for Males and Females at Aged 1 to 90 .

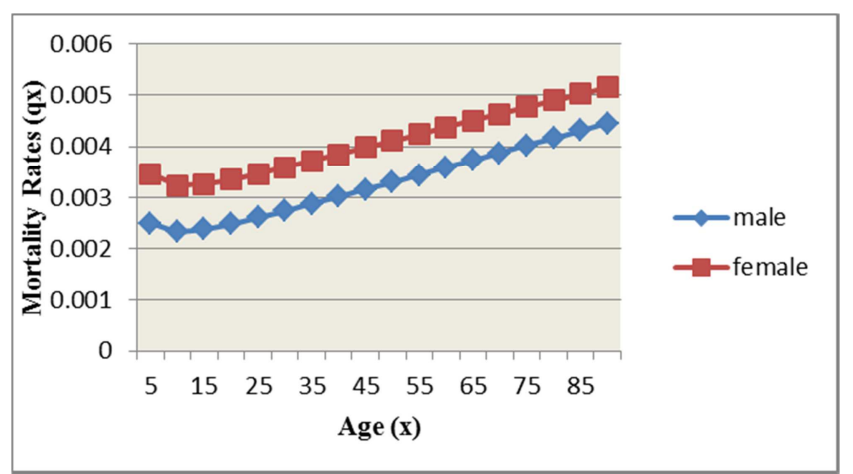

Figure 2. Graph Mortality Rate against Age for Males and Females at Aged 5 to 90 .

Table 3. Results for the Fitted HP Mortality Rates of Males and Females Aged 1-90 for Year 2000, 2003 and 2012.

\begin{tabular}{lllllll}
\hline \multirow{2}{*}{ Age (x) } & Male & \multicolumn{5}{l}{ Female } \\
\cline { 2 - 7 } & $\mathbf{2 0 0 0}$ & $\mathbf{2 0 0 3}$ & $\mathbf{2 0 1 2}$ & $\mathbf{2 0 0 0}$ & $\mathbf{2 0 0 3}$ & $\mathbf{2 0 1 2}$ \\
\hline 1 & 0.004832 & 0.004215 & 0.003454 & 0.006028 & 0.005821 & 0.004482 \\
5 & 0.002493 & 0.002142 & 0.0018 & 0.003461 & 0.003378 & 0.00263 \\
10 & 0.00233 & 0.00201 & 0.001701 & 0.003234 & 0.003172 & 0.00249 \\
15 & 0.002379 & 0.002064 & 0.001751 & 0.00326 & 0.003207 & 0.002532 \\
20 & 0.002481 & 0.002164 & 0.001838 & 0.00335 & 0.003302 & 0.002619 \\
25 & 0.002604 & 0.002281 & 0.001939 & 0.003463 & 0.003419 & 0.002722 \\
30 & 0.002735 & 0.002407 & 0.002047 & 0.003587 & 0.003546 & 0.002832 \\
35 & 0.002872 & 0.002538 & 0.002158 & 0.003716 & 0.003677 & 0.002945 \\
40 & 0.003012 & 0.002671 & 0.002272 & 0.003847 & 0.003811 & 0.00306 \\
45 & 0.003153 & 0.002807 & 0.002387 & 0.003979 & 0.003945 & 0.003175 \\
50 & 0.003296 & 0.002944 & 0.002503 & 0.004111 & 0.004079 & 0.00329 \\
55 & 0.003439 & 0.003081 & 0.00262 & 0.004243 & 0.004213 & 0.003404 \\
60 & 0.003584 & 0.00322 & 0.002737 & 0.004375 & 0.004347 & 0.003519 \\
65 & 0.003728 & 0.00336 & 0.002855 & 0.004506 & 0.00448 & 0.003633 \\
70 & 0.003874 & 0.0035 & 0.002974 & 0.004637 & 0.004612 & 0.003746 \\
75 & 0.00402 & 0.003641 & 0.003093 & 0.004767 & 0.004744 & 0.003859 \\
80 & 0.004166 & 0.003783 & 0.003213 & 0.004897 & 0.004876 & 0.003971 \\
85 & 0.004312 & 0.003926 & 0.003333 & 0.005026 & 0.005006 & 0.004083 \\
90 & 0.004459 & 0.004069 & 0.003454 & 0.005154 & 0.005137 & 0.004195 \\
\hline
\end{tabular}

Table 3 gives the fitted Heligman-Pollard mortality rates for 2000, 2003 and 2012 aged 1 to 90 for both sexes and the results clearly shows that mortality rates are higher in females than in males. And also males and females aged 10 experiences the lowest mortality rates while it also shows an increasing trend from ages 15-90 it also show that as year increase mortality rate decreases for both sexes.

In forecasting future mortality rates the equation (31) is employed and each of the parameters is forecasted. Figure 3 show time series the plots of the forecasted estimates of HP model parameters for males and females respectively from 2017-2036. After each parameter is being forecasted, the forecasted mortality rates are then obtained by plugging these forecasted parameters into (1). From the figures below the forecast plot for male (right) and female (left) from 20172036 with no outliers.

In forecasting the mortality rates of the HP model, ARIMA $(0,1,0)$ was used but to avoid uncertainty which may occur in lengthy forecast only forecasted estimates for 2017, 2018, 2019, 2020 and 2025 was plugged back into the HP function and the mortality rates for these periods were obtained.

Figures 4 and 5 give the fitted (2012) and forecasted (2017) mortality rates for males and females and our results show that mortality rate decreases as time is increasing from 2012-2017 but increases with age from age 15-90 while males and females aged 10-14 experienced lowest mortality rate.

\subsection{Lee-Carter Model}

After applying the techniques in, we arrived at the results. Parameter $\alpha_{x}$ which denotes the empirical average (overtime) pattern of the logarithm mortality rate describing the overall mean of the age profile. Estimated as

$$
\alpha_{x}=\frac{1}{T} \sum_{t=1}^{n} \ln m_{x, t}
$$


Table 4. Results for the estimated $\alpha_{x}$ Parameter.

\begin{tabular}{llll}
\hline $\boldsymbol{\alpha}_{\boldsymbol{x}}=$ & & & \\
\hline Age $(\boldsymbol{x})$ & Male $\left(\boldsymbol{\alpha}_{\boldsymbol{x}}\right)$ & Female $\left(\boldsymbol{\alpha}_{\boldsymbol{x}}\right)$ & Sex-Combined $\left(\boldsymbol{\alpha}_{\boldsymbol{x}}\right)$ \\
\hline$<1$ & 4.470697 & 4.469399 & 5.19455 \\
$1-4$ & 2.694275 & 2.744592 & 3.413021 \\
$5-9$ & 1.334863 & 1.508956 & 2.125205 \\
$10-14$ & 0.6116 & 0.693147 & 1.372504 \\
$15-19$ & 1.257843 & 1.465051 & 2.066315 \\
$20-24$ & 1.74843 & 1.794199 & 2.46771 \\
$25-29$ & 1.929565 & 2.087467 & 2.706283 \\
$30-34$ & 2.144681 & 2.238204 & 2.886165 \\
$35-39$ & 2.360288 & 2.372719 & 3.06009 \\
$40-44$ & 2.498313 & 2.375469 & 3.133026 \\
$45-49$ & 2.625829 & 2.401549 & 3.214772 \\
$50-54$ & 2.844941 & 2.620417 & 3.436126 \\
$55-59$ & 3.082311 & 2.879376 & 3.686204 \\
$60-64$ & 3.50328 & 3.467577 & 4.1923 \\
$65-69$ & 3.888533 & 3.927919 & 4.622491 \\
$70-74$ & 4.33014 & 4.436667 & 5.109158 \\
$75-79$ & 4.777721 & 4.922149 & 5.588082 \\
$80-84$ & 5.206561 & 5.392016 & 6.05011 \\
$85+$ & 5.644895 & 5.839058 & 6.505756 \\
\hline
\end{tabular}

Table 4 and figure 6 give the Results for the estimated $\alpha_{x}$ Parameter and general pattern of mortality by age. From the results above we see that $\alpha_{x}$ is decreasing from less than 1 to 14 years for both males and females, implying a decline in mortality at childhood ages for both sexes but from 15 to 85 and above mortality seems to have risen, thus this shows an upward trend in mortality for both females in that age group. However, it is also observed that males and females at ages

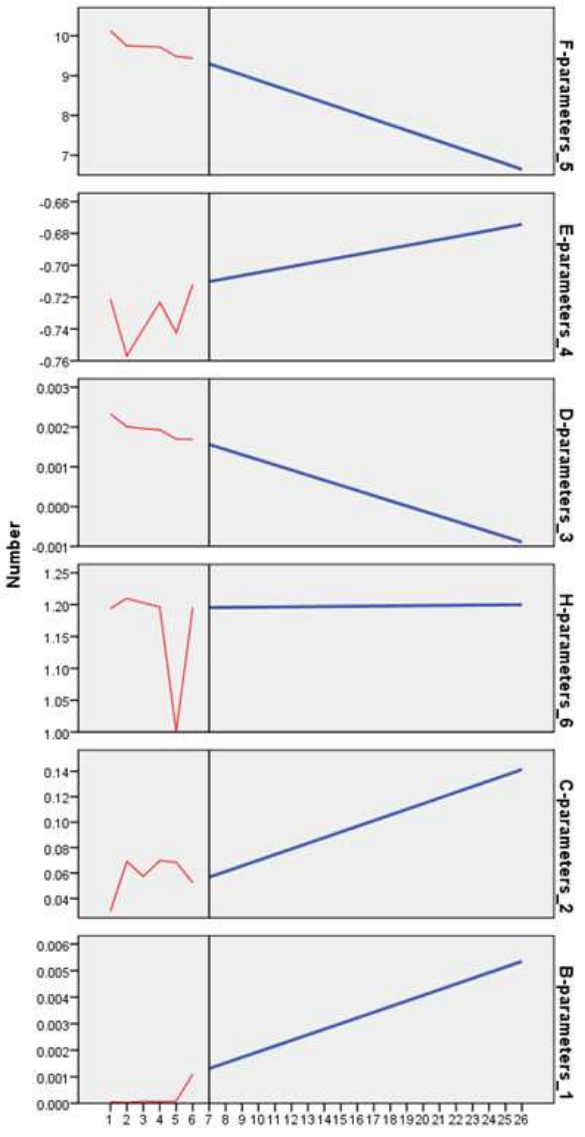

Date

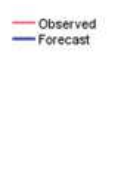

10-14 experiences the lowest mortality rate compared to other age groups. So therefore, in comparing both sexes it is observed that at infant age females seem to have a lower mortality rate than males except from ages 1 to 39 where females seem to have a higher mortality rates than males. It is also observed that males in the age group 40 to 64 have a higher mortality rate than their female counterparts and from 65 to 85 and above, it is otherwise.
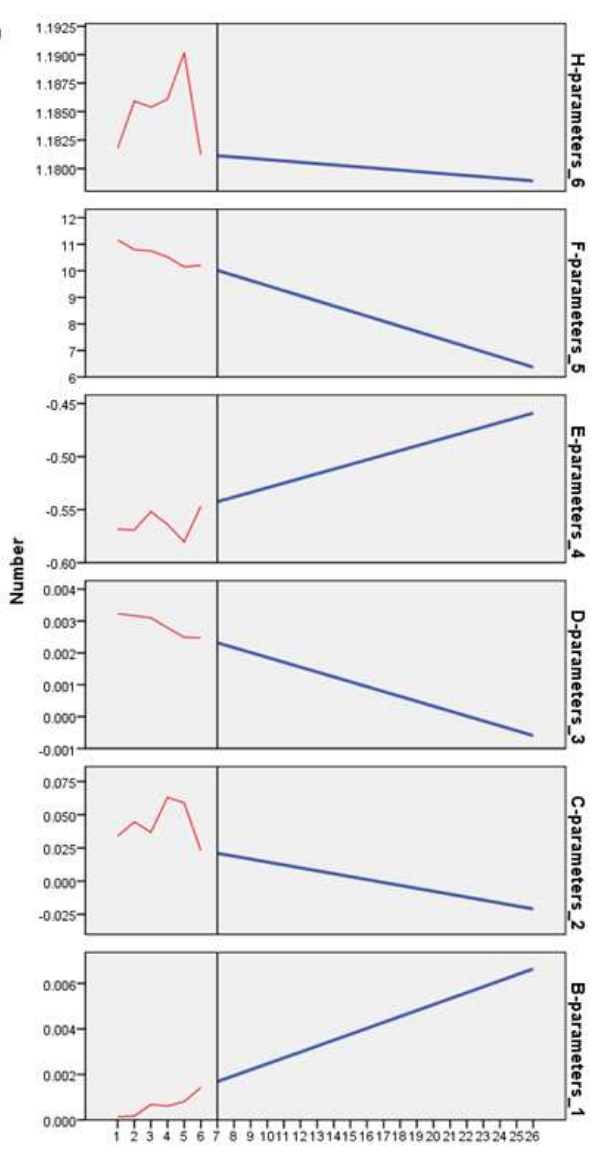

Date

Figure 3. Forecast Plots of the HP Parameters for Males and Females from 2017-2036. 


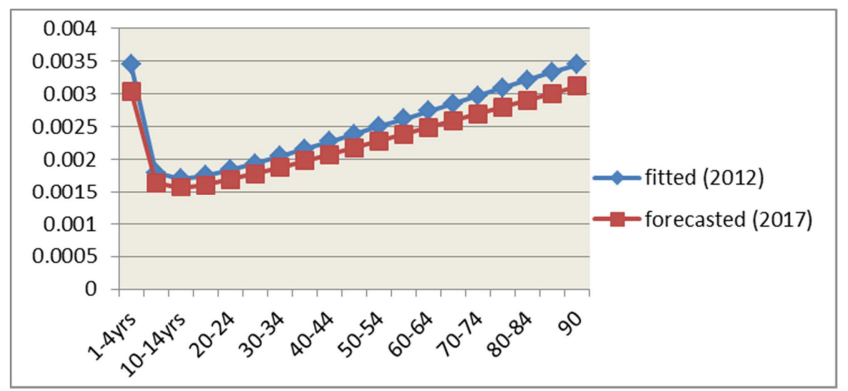

Figure 4. Fitted and Forecasted Mortality Rates for Males.

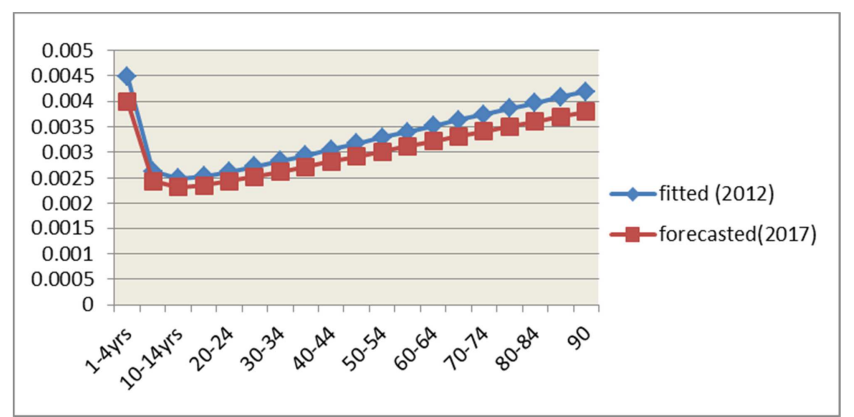

Figure 5. Fitted and Forecasted Mortality Rates for Females.

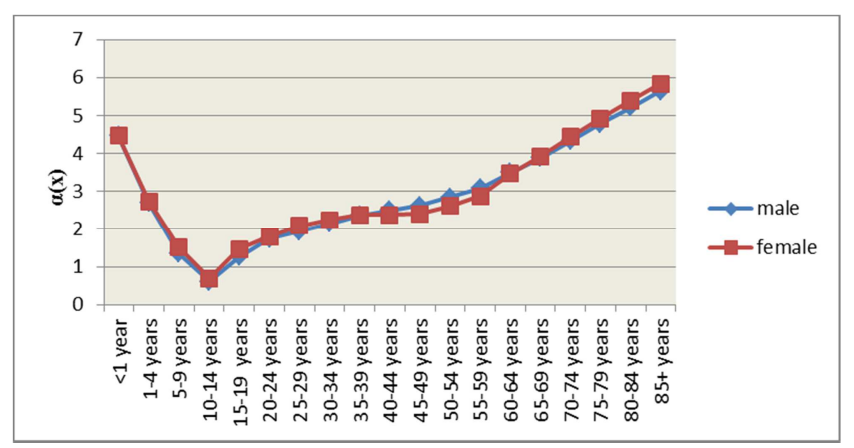

Figure 6. General Pattern of Mortality by Age.

Parameter $\beta_{x}$ denote the sensitivity rate to change at agex to the time trend i.e (it represents the relative rate at which an individual at agex will respond to change in the overall level of mortality overtime). It tells us whether change at a particular age group is faster or slower than the trend itself. Parameter $\beta_{x}$ is derived from the first vector of the agecomponent matrix $\left(\beta_{x}=U_{x 1}\right)$.

Table 5 gives the Results for the estimated $\beta_{x}$ Parameter showing the tendency of mortality at a particular age to change as the general mortality level changes. Therefore, if $\beta_{x}$ is large the mortality rate at that age group will be fluctuant compared to the general level of change in mortality. This results show that persons aged 75-79 have amore fluctuant mortality pattern than other age groups for sex-combined. Where we compare mortality rates in respect to sex, males aged 85 and above have a more fluctuant mortality pattern than females in the same age group. Female persons aged 10-14 seem to have a more fluctuant mortality than their male counterparts as illustrated in figure 7 below. The high values of $\left(\beta_{x}\right)$ indicate improvement in mortality rate at these ages, while the negative values at some ages indicate that mortality rate is increasing.

Table 5. Results for the estimated $\beta_{x}$ Parameter.

\begin{tabular}{llll}
\hline $\boldsymbol{\beta}_{\boldsymbol{x}}=$ & & & \\
\hline Age $(\boldsymbol{x})$ & Male $\left(\boldsymbol{\beta}_{\boldsymbol{x}}\right)$ & Female $\left(\boldsymbol{\beta}_{\boldsymbol{x}}\right)$ & Sex-Combined $\left(\boldsymbol{\beta}_{\boldsymbol{x}}\right)$ \\
\hline$<1$ & 0.01225 & -0.39112 & 0.154193 \\
$1-4$ & 0.044856 & -0.52652 & 0.246184 \\
$5-9$ & -0.10906 & -0.35128 & -0.08248 \\
$10-14$ & -0.21758 & 0 & -0.38039 \\
$15-19$ & -0.12004 & -0.19904 & -0.1361 \\
$20-24$ & -0.04766 & -0.25511 & 0.015931 \\
$25-29$ & -0.02128 & -0.36888 & 0.072244 \\
$30-34$ & 0.010251 & -0.29271 & 0.10989 \\
$35-39$ & 0.041756 & -0.14183 & 0.133028 \\
$40-44$ & 0.062002 & -0.06865 & 0.128363 \\
$45-49$ & 0.080909 & -0.10965 & 0.171272 \\
$50-54$ & 0.113145 & -0.11474 & 0.202528 \\
$55-59$ & 0.14829 & -0.09903 & 0.236891 \\
$60-64$ & 0.21039 & -0.13307 & 0.281943 \\
$65-69$ & 0.267148 & -0.12563 & 0.306167 \\
$70-74$ & 0.332288 & -0.10572 & 0.310655 \\
$75-79$ & 0.398276 & -0.09044 & 0.316669 \\
$80-84$ & 0.461509 & -0.07247 & 0.316134 \\
$85+$ & 0.526094 & -0.0451 & 0.312169 \\
\hline
\end{tabular}

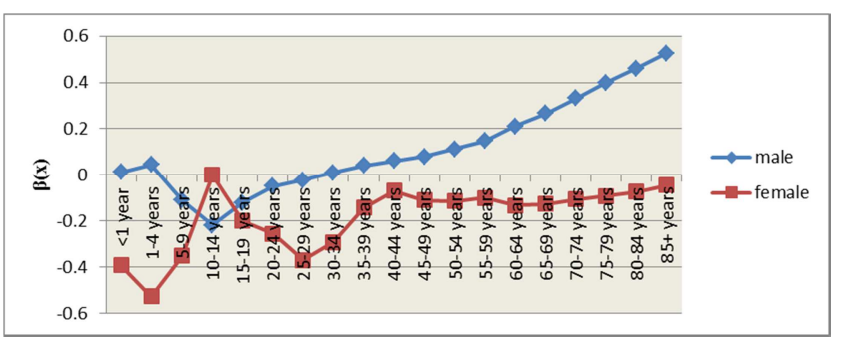

Figure 8. Relative space of change in Mortality by Age.

Parameter $k_{t}$ represents the varying time trend index of general mortality level. It is also referred to as the mortality index capturing the main time trend on the logarithm scale in mortality rates for all ages. $k_{t}$ is derived from the first vector of the time-component matrix and the first singular vector value $\left(k_{t}=L_{1} V_{t 1}\right)$. Table 6 gives Results for the estimated $k_{t}$ Parameter our findings show an upward trend in mortality from 2000-2002 and a slight fall in mortality in 2003 for both males and females. It also shows a downward trend in mortality from 2004 to 2011 and from 2012 to 2016 shows that the mortality pattern was a fluctuating one. Comparing both sexes our results show that males have a higher mortality rate than females from 2000 to 2009 while from 2010-2016 reverse was the case. Males recorded their lowest mortality rates in 2014 while females recorded their lowest mortality rates in 2000. Figure 9 captures the main time trend on the logarithmic scale in death rates at all ages. Therefore, when the values of $k_{t}$ decreases overtime it signifies a fall in mortality trend as the periods increase. The high values of $k_{t}$ indicates there is no improvement in mortality rate.

Table 6. Results for the estimated $k_{t}$ Parameter.

\begin{tabular}{llll}
\hline $\boldsymbol{k}_{\boldsymbol{t}}=$ & & & \\
\hline Year & Male $\left(\boldsymbol{k}_{\boldsymbol{t}}\right)$ & Female $\left(\boldsymbol{k}_{\boldsymbol{t}}\right)$ & Sex-Combined $\left(\boldsymbol{k}_{\boldsymbol{t}}\right)$ \\
\hline 2000 & 0.062259 & -0.33963 & 0.146856 \\
2001 & 0.064968 & -0.33072 & 0.153038 \\
\hline
\end{tabular}




\begin{tabular}{llll}
\hline $\boldsymbol{k}_{\boldsymbol{t}}=$ & & & \\
\hline Year & Male $\left(\boldsymbol{k}_{\boldsymbol{t}}\right)$ & Female $\left(\boldsymbol{k}_{\boldsymbol{t}}\right)$ & Sex-Combined $\left(\boldsymbol{k}_{\boldsymbol{t}}\right)$ \\
\hline 2002 & 0.070153 & -0.2936 & 0.166179 \\
2003 & 0.065728 & -0.27821 & 0.113696 \\
2004 & 0.067055 & -0.23142 & 0.139199 \\
2005 & 0.061578 & -0.16337 & 0.099614 \\
2006 & 0.059962 & -0.09439 & 0.083746 \\
2007 & 0.0554 & -0.04096 & 0.058241 \\
2008 & 0.052758 & -0.0134 & 0.042493 \\
2009 & 0.055608 & 0.049611 & 0.027789 \\
2010 & 0.051779 & 0.093903 & 0.004799 \\
2011 & 0.049508 & 0.155295 & -0.01473 \\
2012 & 0.068599 & 0.164986 & 0.02369 \\
2013 & 0.06678 & 0.245826 & -0.00167 \\
2014 & -0.96984 & 0.27895 & -0.92999 \\
2015 & 0.060935 & 0.366197 & -0.04876 \\
2016 & 0.056775 & 0.430931 & -0.06419 \\
\hline
\end{tabular}

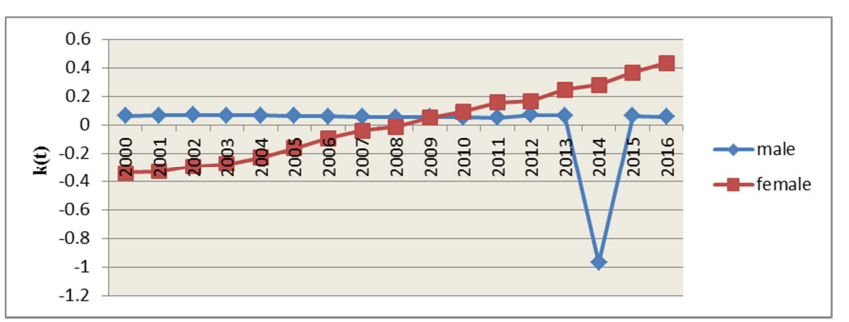

Figure 9. Time Trend for General Mortality.

Figure 10 give the Sum of Square Residuals per Age and Year for Males and Females. From our findings female exhibits a lower sum of squares residual when compared to their male counterpart which can also be a sign of a good fit when both sexes are compared.

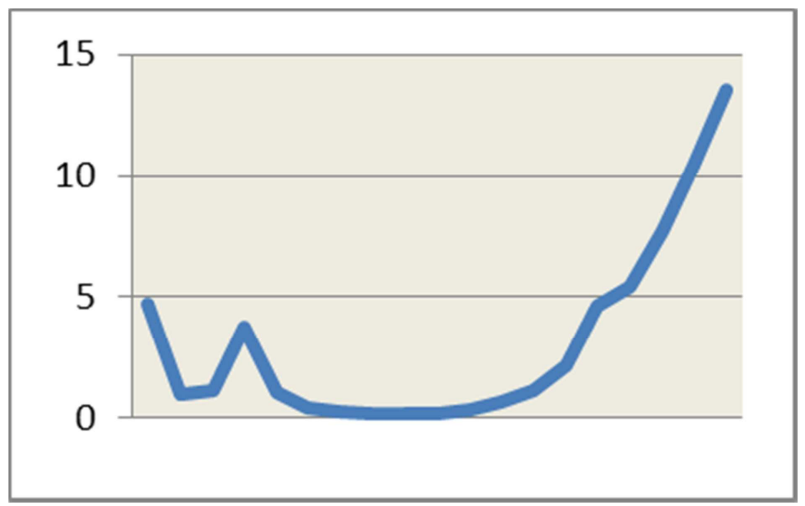

Sum of square Residuals per Age (males).

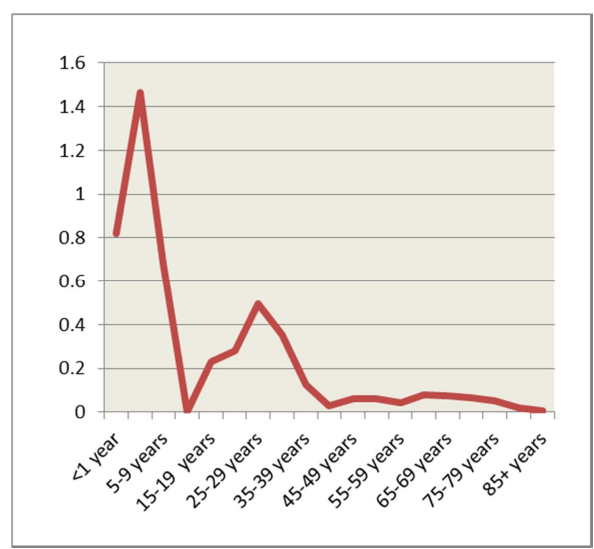

Sum of square Residuals per Age (females).

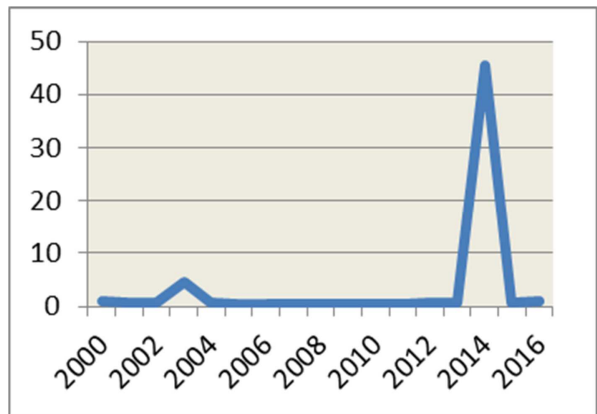

Sum of square Residuals per Year (males).

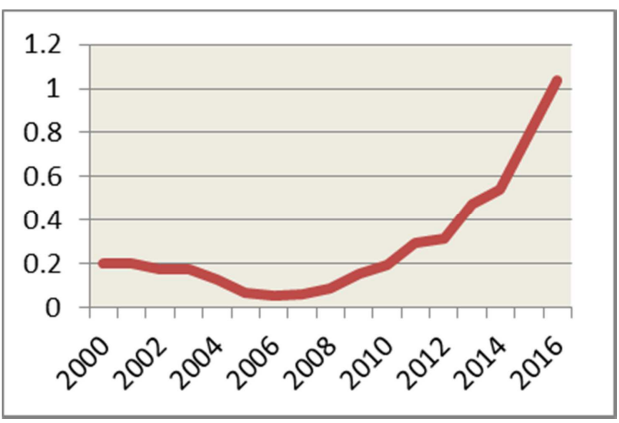

Sum of square Residuals per Year (female).

Figure 10. Sum of Square Residuals per Age and Year for Males and Females.

In forecasting future mortality rates of the Lee-Carter model the equation (31) is employed and only the $k_{t}$ parameter is forecasted. Forecasted parameter estimates are presented in table 7 below.

Table 7. Forecast Table for Mortality Index for Males, Females and Sex-combined from 2017-2036.

\begin{tabular}{|c|c|c|c|c|c|c|c|c|c|}
\hline \multirow{3}{*}{$\begin{array}{l}\text { Sex } \\
\text { Method } \\
\text { Year }\end{array}$} & \multirow{2}{*}{\multicolumn{3}{|c|}{$\begin{array}{l}\text { Male } \\
\text { RWD }\end{array}$}} & \multirow{2}{*}{\multicolumn{3}{|c|}{$\begin{array}{l}\text { Female } \\
\text { RWD }\end{array}$}} & \multicolumn{3}{|c|}{ Sex-Combined } \\
\hline & & & & & & & RWD & & \\
\hline & $\begin{array}{l}\text { Forecast } \\
(k t)\end{array}$ & $\begin{array}{l}\text { Lower LCL } \\
95.0 \%\end{array}$ & $\begin{array}{l}\text { Upper UCL } \\
95.0 \% \\
\end{array}$ & $\begin{array}{l}\text { Forecast } \\
(k t)\end{array}$ & $\begin{array}{l}\text { Lower LCL } \\
95.0 \% \\
\end{array}$ & $\begin{array}{l}\text { Upper UCL } \\
95.0 \%\end{array}$ & Foreca $(k t)$ & $\begin{array}{l}\text { Lower LCL } \\
95.0 \%\end{array}$ & $\begin{array}{l}\text { Upper UCL } \\
95.0 \%\end{array}$ \\
\hline 2017 & 0.066458 & -0.7381 & 0.87099 & 0.512621 & 0.45934 & 0.56590 & -0.07733 & -0.78365 & 0.62899 \\
\hline 2018 & 0.076679 & -1.0611 & 1.214458 & 0.598849 & 0.523501 & 0.674198 & -0.0912 & -1.09069 & 0.90769 \\
\hline 2019 & 0.087438 & -1.30605 & 1.480927 & 0.689616 & 0.597333 & 0.781899 & -0.1058 & -1.32919 & 1.11759 \\
\hline 2020 & 0.098734 & -1.51033 & 1.707797 & 0.784921 & 0.678362 & 0.89148 & -0.12113 & -1.53378 & 1.29152 \\
\hline 2021 & 0.110569 & -1.68842 & 1.909556 & 0.884764 & 0.765628 & 1.003901 & -0.13719 & -1.71658 & 1.442201 \\
\hline 2022 & 0.122942 & -1.84775 & 2.093633 & 0.989146 & 0.858638 & 1.119654 & -0.15398 & -1.88411 & 1.576156 \\
\hline 2023 & 0.135852 & -1.99274 & 2.264442 & 1.098066 & 0.957101 & 1.239031 & -0.1715 & -2.04025 & 1.697261 \\
\hline
\end{tabular}




\begin{tabular}{|c|c|c|c|c|c|c|c|c|c|}
\hline \multirow{3}{*}{$\begin{array}{l}\text { Sex } \\
\text { Method } \\
\text { Year }\end{array}$} & \multirow{2}{*}{\multicolumn{3}{|c|}{$\begin{array}{l}\text { Male } \\
\text { RWD }\end{array}$}} & \multirow{2}{*}{\multicolumn{3}{|c|}{$\begin{array}{l}\text { Female } \\
\text { RWD }\end{array}$}} & \multicolumn{3}{|c|}{ Sex-Combined } \\
\hline & & & & & & & RWD & & \\
\hline & $\begin{array}{l}\text { Forecast } \\
(k t)\end{array}$ & $\begin{array}{l}\text { Lower LCL } \\
95.0 \%\end{array}$ & $\begin{array}{l}\text { Upper UCL } \\
95.0 \%\end{array}$ & $\begin{array}{l}\text { Forecast } \\
(k t)\end{array}$ & $\begin{array}{l}\text { Lower LCL } \\
95.0 \%\end{array}$ & $\begin{array}{l}\text { Upper UCL } \\
95.0 \%\end{array}$ & Foreca $(k t)$ & $\begin{array}{l}\text { Lower LCL } \\
95.0 \%\end{array}$ & $\begin{array}{l}\text { Upper UCL } \\
95.0 \%\end{array}$ \\
\hline 2024 & 0.149301 & -2.12626 & 2.424859 & 1.211525 & 1.060827 & 1.362222 & -0.18975 & -2.18753 & 1.80804 \\
\hline 2025 & 0.163287 & -2.25031 & 2.576882 & 1.329521 & 1.169682 & 1.48936 & -0.20873 & -2.32770 & 1.910247 \\
\hline 2026 & 0.177812 & -2.36634 & 2.721963 & 1.452056 & 1.283571 & 1.620541 & -0.22843 & -2.46203 & 2.005158 \\
\hline 2027 & 0.192874 & -2.47545 & 2.861203 & 1.57913 & 1.402421 & 1.755838 & -0.24887 & -2.59149 & 2.093738 \\
\hline 2028 & 0.208474 & -2.57850 & 2.995453 & 1.710741 & 1.526175 & 1.895307 & -0.27004 & -2.71682 & 2.176735 \\
\hline 2029 & 0.224613 & -2.67617 & 3.125392 & 1.846891 & 1.654789 & 2.038994 & -0.29194 & -2.83863 & 2.254745 \\
\hline 2030 & 0.241289 & -2.76899 & 3.25157 & 1.98758 & 1.788225 & 2.186934 & -0.31457 & -2.95739 & 2.328251 \\
\hline 2031 & 0.258503 & -2.85743 & 3.37444 & 2.132806 & 1.926455 & 2.339157 & -0.33793 & -3.07351 & 2.39765 \\
\hline 2032 & 0.276255 & -2.94187 & 3.494381 & 2.282571 & 2.069453 & 2.49569 & -0.36202 & -3.18732 & 2.463276 \\
\hline 2033 & 0.294545 & -3.02262 & 3.611713 & 2.436875 & 2.217197 & 2.656552 & -0.38684 & -3.29909 & 2.525409 \\
\hline 2034 & 0.313373 & -3.09996 & 3.726711 & 2.595716 & 2.369670 & 2.821763 & -0.41239 & -3.40907 & 2.584291 \\
\hline 2035 & 0.332739 & -3.17413 & 3.83961 & 2.759096 & 2.526856 & 2.991337 & -0.43867 & -3.51746 & 2.640127 \\
\hline 2036 & 0.352643 & -3.24533 & 3.950617 & 2.927015 & 2.688741 & 3.165289 & -0.46568 & -3.62445 & 2.693100 \\
\hline
\end{tabular}

Table 7 gives the forecast table for mortality index for males, females and sex-combined from 2017-2036 during the period where actual data is not available. It also show the predicted values from the fitted model with $95 \%$ prediction line indicating the upper and lower confidence limits. Forecasted parameters were later plugged back into equation (8) to get the forecasted log mortality rates. Figure 11 shows the forecast plot mortality index for sex-combined. The forecast of the mortality time trend show a gradual decline in mortality from 2017-2036.

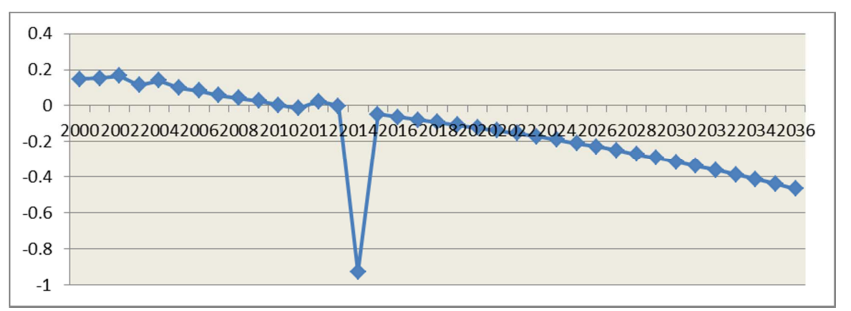

Figure 11. Forecast Plot Mortality Index for Sex-Combined from 2017-2036.

Figure 11 shows the forecast plot mortality index for sexcombined. The forecast of the mortality time trend show a gradual decline in mortality from 2017-2036.

Figures 12 and 13 illustrate the log mortality rates (actual 2016, fitted 2016, forecast 2025 \& 2036) for males and females respectively. From our findings male in ages 30 to $85+$ will experience a slight increase in mortality rate in 2025 and 2036 but it is otherwise for females. It also shows that at older ages as female mortality rate falls with time male mortality increases. Also forecasted log mortality rates in females are lower than in males as shown below.

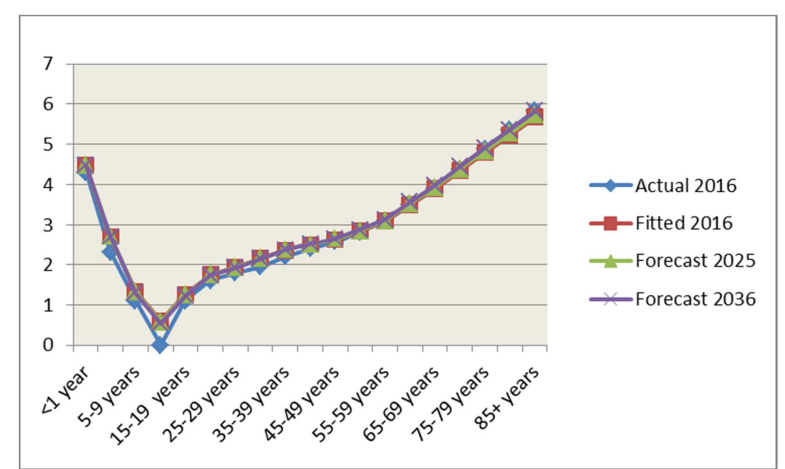

Figure 12. Log Mortality Rates (actual 2016, fitted 2016, forecast 2025 \& 2030) for Males.

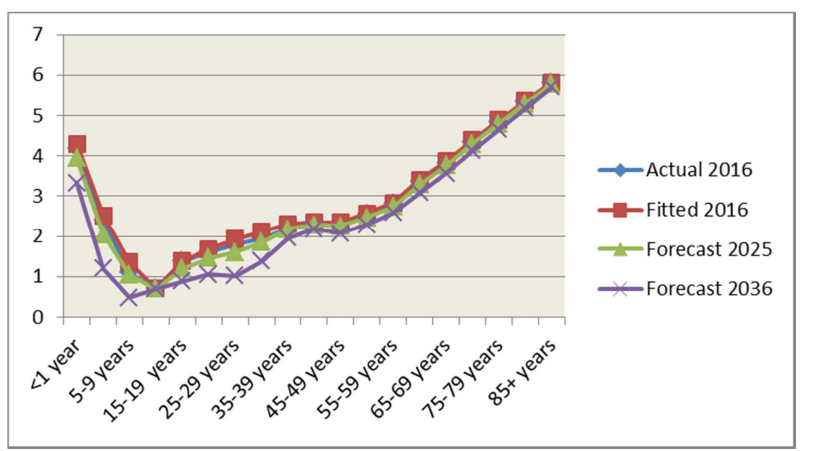

Figure 13. Log Mortality Rates (actual 2016, fitted 2016, forecast 2025 \& 2030) for Females.

\subsection{Forecast Results}

Table 8. Model Description.

\begin{tabular}{llll}
\hline Model Type & & & \\
\hline \multirow{4}{*}{ Model ID } & Male HP & Parameter_1 & ARIMA $(0,1,0)$ \\
& Male LC & Parameter_2 & ARIMA $(0,1,0)$ \\
& Female HP & Parameter_3 & ARIMA $(0,1,0)$ \\
& Female LC & Parameter_4 & ARIMA $(0,1,0)$ \\
\hline
\end{tabular}




\subsubsection{Diagnostics}

Table 9. Model Fit HP.

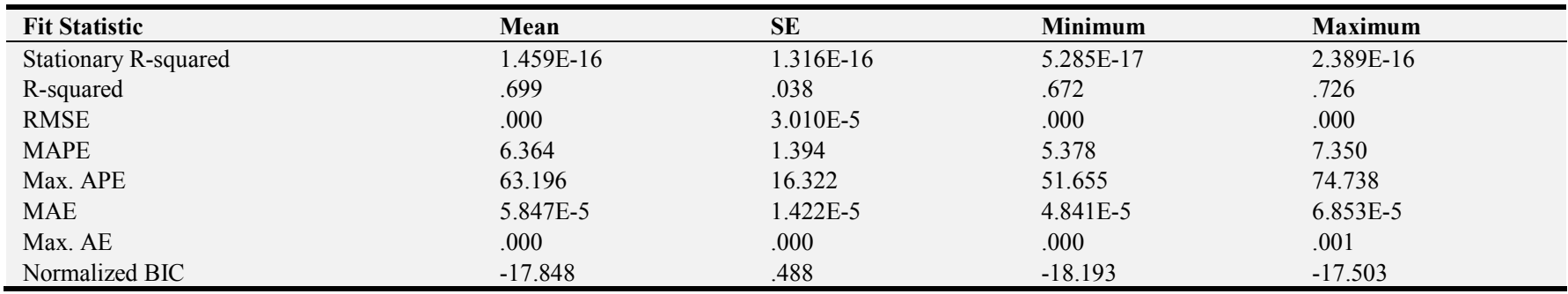

Table 9. Continued.

\begin{tabular}{|c|c|c|c|c|c|c|c|}
\hline \multirow{2}{*}{ Fit Statistic } & \multicolumn{7}{|l|}{ Percentile } \\
\hline & 5 & 10 & 25 & 50 & 75 & 90 & 95 \\
\hline Stationary R-squared & $5.285 \mathrm{E}-17$ & $5.285 \mathrm{E}-17$ & $5.285 \mathrm{E}-17$ & $1.459 \mathrm{E}-16$ & $2.389 \mathrm{E}-16$ & $2.389 \mathrm{E}-16$ & $2.389 \mathrm{E}-16$ \\
\hline R-squared & .672 & .672 & .672 & 699 & .726 & .726 & .726 \\
\hline RMSE & .000 & .000 & .000 & .000 & .000 & .000 & .000 \\
\hline MAPE & 5.378 & 5.378 & 5.378 & 6.364 & 7.350 & 7.350 & 7.350 \\
\hline Max. APE & 51.655 & 51.655 & 51.655 & 63.196 & 74.738 & 74.738 & 74.738 \\
\hline MAE & $4.841 \mathrm{E}-5$ & $4.841 \mathrm{E}-5$ & $4.841 \mathrm{E}-5$ & 5.847E-5 & $6.853 \mathrm{E}-5$ & $6.853 \mathrm{E}-5$ & $6.853 \mathrm{E}-5$ \\
\hline Normalized BIC & -18.193 & -18.193 & -18.193 & -17.848 & -17.503 & -17.503 & -17.503 \\
\hline
\end{tabular}

Table 9 gives the model fit of the Heligman-Pollard model. From our findings we see that the fit statistic with the lowest mean is the root mean square error (RMSE) which is not significant with a mean absolute percentage error (MAPE) 6.364 and mean absolute error (MAE) 5.847E-5.

Table 10. Model Fit LC

\begin{tabular}{lllll}
\hline Fit Statistic & Mean & SE & Minimum & Maximum \\
\hline Stationary R-squared & $6.706 \mathrm{E}-17$ & $2.705 \mathrm{E}-16$ & $-1.242 \mathrm{E}-16$ & $2.584 \mathrm{E}-16$ \\
R-squared & .803 & .028 & .783 & .824 \\
RMSE & .650 & .028 & .630 & .670 \\
MAPE & 24.545 & 2.712 & 22.627 & 26.462 \\
Max. APE & 123.373 & 24.588 & 105.987 & 140.760 \\
MAE & .433 & .032 & .410 & .456 \\
Max. AE & 1.924 & .117 & 1.841 & 2.006 \\
Normalized BIC & -.701 & .086 & -.762 & -.640 \\
\hline
\end{tabular}

Table 10. Continued.

\begin{tabular}{|c|c|c|c|c|c|c|c|}
\hline \multirow{2}{*}{ Fit Statistic } & \multicolumn{7}{|l|}{ Percentile } \\
\hline & 5 & 10 & 25 & 50 & 75 & 90 & 95 \\
\hline Stationary R-squared & $-1.242 \mathrm{E}-16$ & $-1.242 \mathrm{E}-16$ & $-1.242 \mathrm{E}-16$ & $6.706 \mathrm{E}-17$ & $2.584 \mathrm{E}-16$ & $2.584 \mathrm{E}-16$ & $2.584 \mathrm{E}-16$ \\
\hline R-squared & .783 & .783 & .783 & .803 & .824 & .824 & .824 \\
\hline RMSE & .630 & .630 & .630 & .650 & .670 & .670 & 670 \\
\hline MAPE & 22.627 & 22.627 & 22.627 & 24.545 & 26.462 & 26.462 & 26.462 \\
\hline Max. APE & 105.987 & 105.987 & 105.987 & 123.373 & 140.760 & 140.760 & 140.760 \\
\hline Max. AE & 1.841 & 1.841 & 1.841 & 1.924 & 2.006 & 2.006 & 2.006 \\
\hline Normalized BIC & -.762 & -.762 & -.762 & -.701 & -.640 & -.640 & -.640 \\
\hline
\end{tabular}

Table 10 gives the model fit Lee-Carter model, the diagnostic test for standardized residuals (Ljung-Box) for each model is conducted to ascertain the robustness of the estimated models. From our results we see that the fit statistic with the lowest mean is the mean absolute error (MAE) which is 0.433 , root mean square error (RMSE) 0.650 and man absolute percentage error (MAPE) 24.545.

\subsubsection{Forecast Performance}

Using the loss functions MAE and MAPE in equation (39) and (40) respectively the predictive abilities of the models are presented in Table 11. 
Table 11. Model Statistics.

\begin{tabular}{lllll}
\hline \multirow{2}{*}{ Model } & \multirow{2}{*}{ Number of Predictors } & Model Fit statistics & \\
\cline { 2 - 5 } & & Stationary R-squared & R-squared & .726 \\
\hline Male H-P & 0 & $2.389 \mathrm{E}-16$ & .783 & .000 \\
Male LC & 0 & $2.584 \mathrm{E}-16$ & .670 \\
Female H-P & 0 & $5.285 \mathrm{E}-17$ & .672 & .000 \\
Female LC & 0 & $-1.242 \mathrm{E}-16$ & .824 & .630 \\
\hline
\end{tabular}

Table 11. Continued.

\begin{tabular}{|c|c|c|c|c|c|c|c|}
\hline \multirow{2}{*}{ Model } & \multicolumn{3}{|c|}{ Model Fit statistics } & \multicolumn{3}{|c|}{ Ljung-Box Q (18) } & \multirow{2}{*}{ Number of Outliers } \\
\hline & MAPE & MAE & Normalized BIC & Statistics & DF & Sig. & \\
\hline Male H-P & 7.350 & $4.841 \mathrm{E}-5$ & -18.193 & . & 0 &. & 0 \\
\hline Male LC & 26.462 & .456 & -.640 & . & 0 & . & 0 \\
\hline Female H-P & 5.378 & $6.853 \mathrm{E}-5$ & -17.503 & . & 0 & . & 0 \\
\hline Female LC & 22.627 & .410 & -.762 & . & 0 & . & 0 \\
\hline
\end{tabular}

Table 11 compares the results of the models using ARIMA $(0,1,0)$ model. Looking at the error statistics the model with the smallest mean absolute percentage error (MAPE) during estimation period is the female HP model. Therefore mean absolute error (MAE) for the HP model or both sexes indicates that the model forecasts are able to account for much of the variability of the data used for the forecast.

Table 12. ARIMA Model Parameters.

\begin{tabular}{|c|c|c|c|c|c|c|c|}
\hline & & & & Estimate & SE & t & Sig. \\
\hline Male HP & Male HP & No Transformation & $\begin{array}{l}\text { Constant } \\
\text { Difference }\end{array}$ & $\begin{array}{l}1.006 \mathrm{E}-5 \\
1\end{array}$ & $2.438 \mathrm{E}-5$ & .413 & .685 \\
\hline Male LC & Male LC & No Transformation & $\begin{array}{l}\text { Constant } \\
\text { Difference }\end{array}$ & $\begin{array}{l}.070 \\
1\end{array}$ & .158 & .443 & .664 \\
\hline Female HP & Female HP & No Transformation & $\begin{array}{l}\text { Constant } \\
\text { Difference }\end{array}$ & $\begin{array}{l}9.508 \mathrm{E}-6 \\
1\end{array}$ & $3.441 \mathrm{E}-5$ & .276 & .786 \\
\hline Female LC & Female LC & No Transformation & $\begin{array}{l}\text { Constant } \\
\text { Difference }\end{array}$ & $\begin{array}{l}.102 \\
1\end{array}$ & .149 & .684 & .503 \\
\hline
\end{tabular}

\subsection{Comparison of the Models}

We have compared two models by choosing the best fitting model from both models. Specifically, we have compared the Heligman and Pollard's law for $q_{x}$ and Lee and Carter model for $m_{x}$. We have also obtained the values of the root mean square error (RMSE), Mean absolute error (MAE), mean absolute percentage error (MAPE), and $R^{2}$. The value of $R^{2}$ has been obtained as 1 minus the proportion of the variance that remains unexplained, because if we calculate it directly as a percentage of explained variance, in some cases it exceeded this can happen when the models are not linear. Therefore the comparison both models will be based on the mean absolute percentage error (MAPE). Comparing their overall fit, the HP model seems to fit better to the Nigeria data which is a surprising result since the HP model is supposed to be worse in forecasting than the LC model.

\subsection{Life Expectancy}

The number of years a person at age $\mathrm{x}$ is expected to live is derived from (43). From the Abridged life table presented in the Appendix, Table 13 shows the life expectancy table for fitted (2000) and forecasted (2017-2020) mortality rates for males and femalesgiven the life expectancy at birth and age 75, fitted 2000 and forecasted 2017-2020 for males and females. From our results we see that males experience higher life expectancy than females which is not a striking result due to the mortality rates experienced by both sexes

Table 13. Life Expectancy Table Fitted (2000) and Forecasted (2017-2020) for Males and Females.

\begin{tabular}{|c|c|c|c|c|}
\hline & Male & & Femal & \\
\hline & $e_{0}$ & $e_{75}$ & $e_{0}$ & $e_{75}$ \\
\hline Fitted HP (2000) & 77.28 & 12.18 & 74.55 & 12.13 \\
\hline Forecasted (2017-2020) & 65.64 & 11.73 & 58.79 & 11.53 \\
\hline
\end{tabular}

\section{Conclusion}

From our analysis we see that the Lee-Carter model follow the mortality pattern very well for most of the ages except that the fit of the model was better for female data than male according to the sum of square of the residuals. Despite the fluctuating mortality pattern the model was able to fit mortality rates. However the HP seems to overestimate female mortality especially from $15-85+$. Thus, since there are significant difference between the actual rates and the fitted rates one can conclude that the HP model is not the best model for Nigeria 
data. Looking at the overall fit, it is a surprising to see that the HP model performed better than LC considering the fact that the HP model is supposed to be worse in forecasting than the LC model. Although the HP model fitted mortality rates are significantly different from the actual mortality rate the forecast seem to be more reliable based on the (MAPE). The abridged life table shows that male experienced a higher life expectancy and female at infant and late adult ages. To ascertain level and pattern of mortality rate especially in adult, there is also need for the use of statistical model especially the HP model to forecast mortality rate because of it lesser errors as shown in this study and also to improve the understanding of the pattern of general mortality rate and how it affects life expectancy level in the country.

\section{Appendix}

Abridged Life Table for Male (2000)

Number of life tables: 1

Dimension: $19 \times 10$

Age intervals: $[0,1)[1,5)[5,10) \ldots \ldots[75,80)[80,85)$ $[85,+)$

$$
\text { x. intx } m_{x} q_{x} a_{x} l_{x} d_{x} L_{x} T_{x} e_{x}
$$

$\left[\begin{array}{llllllll}0,1) & 0 & 0.0048 & 0.0048 & 0.06 & 1 \mathrm{e}+05 & 48299546 & 7727605\end{array}\right.$ 77.28

$[1,5) 10.00250 .00991 .64995189873957397628059$ 76.65

$[5,10) 50.00230 .01162 .59853111414897947232320$ 73.4

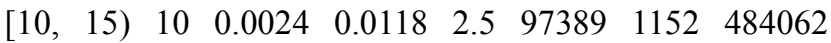
674252669.23

$\left[\begin{array}{llllllll}15,20) & 15 & 0.0025 & 0.0123 & 2.49 & 96238 & 1186 & 478216\end{array}\right.$ 625846465.03

$\left[\begin{array}{lllllllll}20, & 25) & 20 & 0.0026 & 0.0129 & 2.49 & 95051 & 1230 & 472176\end{array}\right.$ 578024860.81

$<\mathrm{NA}>\ldots \ldots \ldots \ldots \ldots \ldots \ldots$

$\left[\begin{array}{lllllllll}75, & 80) & 75 & 0.0042 & 0.0206 & 2.49 & 79268 & 1634 & 392239\end{array}\right.$ 96551512.18

$\left[\begin{array}{lllllllll}80, & 85) & 80 & 0.0043 & 0.0213 & 2.49 & 77634 & 1656 & 384014\end{array}\right.$ 5732767.38

$[85,+) 850.00450 .0222 .497597875978189262189262$ 2.49

Abridged Life Table for Female (2000)

Number of life tables: 1

Dimension: $19 \times 10$

Age intervals: $[0,1)[1,5)[5,10) \ldots \ldots[75,80)[80,85)$ $[85,+)$

$$
\text { x. intx } m_{x} q_{x} a_{x} l_{x} d_{x} L_{x} T_{x} e_{x}
$$

$[0,1) 00.0060 .0060 .071 \mathrm{e}+0560199441745548874.55$ $[1,5) 10.00350 .01371 .519939913673941977356047$ 74.01

$[5,10) 50.00320 .0162 .499803215724862206961850$ 71.02

$\left[\begin{array}{lllllllll}10, & 15) & 10 & 0.0033 & 0.0162 & 2.49 & 96460 & 1560 & 478390\end{array}\right.$
647562967.13

$\left[\begin{array}{llllllll}15,20) & 15 & 0.0034 & 0.0166 & 2.49 & 94900 & 1576 & 470550\end{array}\right.$ 599723963.2

$\left[\begin{array}{lllllllll}20, & 25) & 20 & 0.0035 & 0.0172 & 2.49 & 93324 & 1602 & 462604\end{array}\right.$ 552668959.22

$<\mathrm{NA}>\ldots \ldots \ldots \ldots \ldots \ldots \ldots$

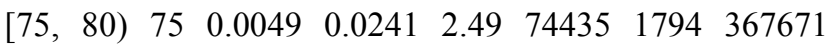
90270412.13

$[80,85) 800.0050 .02482 .49726411803358679535033$ 7.37

[85+) 850.00520 .02542 .497083870838176354176354 2.49

Abridged Life Table for Male (2017-2020)

Number of life tables: 1

Dimension: $19 \times 10$

Age intervals: $[0,1)[1,5)[5,10) \ldots \ldots[75,80)[80,85)$ $[85,+)$

$$
x . \text { int } x m_{x} q_{x} a_{x} l_{x} d_{x} L_{x} T_{x} e_{x}
$$

$\left[\begin{array}{llllllll}0,1) & 0 & 0.0105 & 0.0104 & 0.08 & 1 \mathrm{e}+05 & 104599041 & 6563751\end{array}\right.$ 65.64

$[1,5) 10.00570 .02271 .519895522483902146464709$ 65.33

$[5,10) 50.00550 .0272 .499670726154769686074495$ 62.81

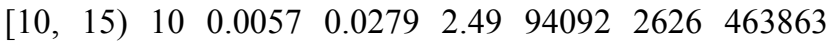
559752759.49

$\left[\begin{array}{lllllllll}15,20) & 15 & 0.0059 & 0.0293 & 2.49 & 91466 & 2681 & 450593\end{array}\right.$ 513366556.13

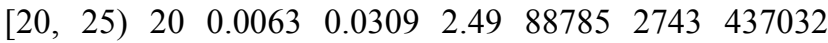
468307252.75

$<\mathrm{NA}>$

$\left[\begin{array}{lllllllll}75,80) & 75 & 0.0103 & 0.0501 & 2.48 & 56937 & 2855 & 277487\end{array}\right.$ 66786111.73

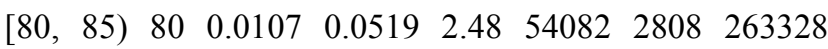
3903757.22

$[85,+) 850.0110 .05372 .485127451274127046127046$ 2.48

Abridged Life Table for Female (2017-2020)

Number of life tables: 1

Dimension: $19 \times 10$

Age intervals: $[0,1)[1,5)[5,10) \ldots \ldots[75,80)[80,85)$ $[85,+)$

$$
\text { x. intx } m_{x} q_{x} a_{x} l_{x} d_{x} L_{x} T_{x} e_{x}
$$

$\left[\begin{array}{lllllllll}0,1) & 0 & 0.0141 & 0.014 & 0.09 & 1 \mathrm{e}+05 & 1401 & 98728 & 5878714\end{array}\right.$ 58.79

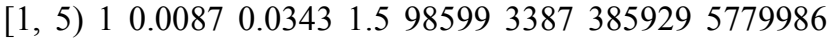
58.62

$[5,10) 50.00840 .04092 .489521238954662545394056$ 56.65

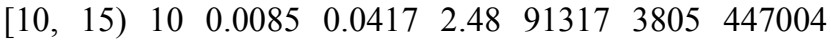
492780353.96

$\left[\begin{array}{llllllll}15,20) & 15 & 0.0088 & 0.0431 & 2.48 & 87512 & 3768 & 428070\end{array}\right.$ 448079951.2

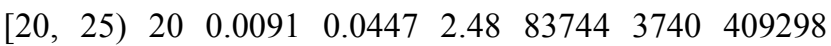


405272848.39

$<$ NA $>$

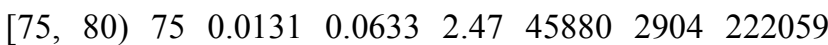
52922211.53

$\left[\begin{array}{llllllll}80,85) & 80 & 0.0134 & 0.0649 & 2.47 & 42975 & 2790 & 207824\end{array}\right.$ 3071637.15

$[85,+) 850.01380 .06652 .4740185401859933999339$ 2.47

\section{References}

[1] Lee R D, Carter L R. (1992). "Modeling and forecasting U.S mortality" Journal of the American Statistical Association, 87: 659-675.

[2] Yahaya. H. U and Tanimu. M Analysis of mortality rate in Nigeria (2016). The International Journal of science and Technoledge (ISSN2321-919x) 80-84.

[3] Angela U. Chukwu [a], and E. O. Oladipupo March 12, (2012)[a] Modelling Adult Mortality in Nigeria: An Analysis Based on the Lee-Carter Model [a] Department of Statistics, University of Ibadan, Nigeria. 3-5.

[4] R. I Ibrahim et.al (2017) forecasting the mortality rates using Lee-Carter model and Heligman-Pollard model (journal of physics: conference series 890012128 .

[5] Wouter van Wel (2015), Mortality Modeling and forecasting using Cross-Validation Techniques. I.

[6] L. Heligman, M. A. and J. H. Pollard (1980) "The age pattern of mortality JIA 107 (1980) 49-80.

[7] L. Vandenberghe ECE133A (Fall 2018) 13. Nonlinear least squares Algorithm (Minpack).
[8] $\mathrm{R}$ interface of the Levenberg-Marquadt nonlinear Leastsquares algorithm. Package "Minpack. Im, Nov 20, (2016) version 1.2-1.

[9] Renshaw E. A, S. Haberman (2006). A cohort based extension to the lee-Carter model for Mortality reduction factors: Insurance; Mathematics and economics 38: 556-70.

[10] Lipkovich, Ilya and Smith, Eric P. (June 2002). Biplot and Singular Value Decomposition Macros for Excel.

[11] Rose Irnawaty Ibrahim Expanding an Abridged Life Table Using the Heligman-Pollard Model. 2008, Volume 24, Number $1,1\{10\}$.

[12] Lee, Ronald and Miller, Timothy (November 2000). Evaluating the Performance of Lee-Carter Mortality Forecasts. 50 .

[13] R. McNown and A. Rogers forecasting cause-specific mortality using time series methods (international journal of forecasting 8: 413-432) 1992.

[14] Ujah I. A. O, Aiseen O. A, Mutihir, J. T Vanderjagt, DJ. Glenwqwand, R, A and Ugwu V., E (2005) factors contributing to maternal mortality in North-central Nigeria: A seventeen-year review (African journal of reproductive health, 9, 27-40.

[15] Li, Siu-Hang and Chan, Wai-Sum (January 2005). The LeeCarter Model for Forecasting Mortality Revisited.

[16] Olsén, Jörgen (July 2005) Modeller och Projektioner för Dödlighetsintensitet - en anpassning till svensk populations data 1970-2004. H. Booth, Rob J. Hyndman, L Tickle and P. de Jong Lee-Carter mortality forecasting: A multi-Country comparison of variants extensions (Demographic research, 15: 289-310, 2006). 